Pure and Applied Mathematics Quarterly

Volume 9, Number 3

$507-546,2013$

\title{
Bergman Kernel and Kähler Tensor Calculus
}

\author{
$\mathrm{Hao} \mathrm{Xu}$
}

\begin{abstract}
Fefferman [22] initiated a program of expressing the asymptotic expansion of the boundary singularity of the Bergman kernel for strictly pseudoconvex domains explicitly in terms of boundary invariants. Hirachi, Komatsu and Nakazawa [30] carried out computations of the first few terms of Fefferman's asymptotic expansion building partly on Graham's work on CR invariants and Nakazawa's work on the asymptotic expansion of the Bergman kernel for strictly pseudoconvex complete Reinhardt domains. In this paper, we prove a formula for coefficients in Nakazawa's asymptotic expansion as explicit summations over strongly connected graphs, and a formula expressing partial derivatives of Kähler metrics (resp. functions) as summations over rooted trees encoding covariant derivatives of curvature tensors (resp. functions). These formulae shall be useful in studying general patterns of Fefferman's asymptotic expansion.
\end{abstract}

Keywords: Bergman kernel, asymptotic expansion, Laplace integral.

\section{INTRODUCTION}

The heat kernel on Riemannian manifolds plays an important role in index theory, general relativity and cosmology. A central object of study is the shorttime expansion of the heat kernel of the Laplacian operator,

$$
K(t, x, x) \sim \sum_{j=0}^{\infty} a_{j}(x) t^{j-d / 2}, \quad t \rightarrow 0+,
$$

where $d$ is the manifold's dimension and the coefficients $a_{j}(x)$ are invariant polynomials of jets of metrics. By H. Weyl's work on the invariants of the orthogonal

Received August 21, 2012. 
group, any invariant polynomial can be formed from Riemannian curvature tensor by covariant differentiations, multiplications and contractions. It is a very difficult problem to put a tensor expressions into a canonical form on a general Riemmanian manifold. While the asymptotic expansion of the heat kernel has important applications in spectral geometry (see [58] for a recent survey), it seems hopeless to detect any meaningful structure of its combinatorially explosive coefficients.

The ubiquitousness of heat kernels can be seen most clearly in Liu's work $[39,40,41]$, where the heat kernel was used as a unifying tool to solve problems in algebra, geometry and topology.

Let $\Omega$ be a (bounded) domain in $\mathbb{C}^{n}$ and $A^{2}(\Omega)$ the Bergman space of holomorphic functions in $L^{2}(\Omega)$. The (unweighted) Bergman kernel of $\Omega$ is a real analytic function given by $K(z)=\sum_{j}\left|\phi_{j}(z)\right|^{2}$ for $z \in \Omega$, where $\left\{\phi_{j}\right\}$ is an arbitrary orthonormal basis of $A^{2}(\Omega)$. In [22], Fefferman initiated a program of studying the Bergman kernel of a strictly pseudoconvex domain as an analogy of the heat kernel, with the time variable $t$ replaced by a defining function of the domain. Fefferman's program opened up the subject $[3,25,27,30]$ and has been extended to conformal geometry; a major recent breakthrough is Alexakis' proof [1] of the Deser-Schwimmer conjecture.

Yau [55, p.679] proposed to classify pseudoconvex domains whose Bergman metrics are Kähler-Einstein. A conjecture of S.-Y. Cheng says that if the Bergman metric of a strictly pseudoconvex domain is Kähler-Einstein, then the domain is biholomorphic to the ball (cf. [23]). The deep works of Cheng-Yau [11] and MokYau [47] showed that on a bounded domain of holomorphy, there exists a unique biholomorphic invariant complete Kähler-Einstein metric with scalar curvature -1 .

The complete asymptotic expansion of the weighted Bergman kernel has been established by Engliš [18] for bounded strictly pseudoconvex domains in $\mathbb{C}^{n}$ with real analytic boundary. For compact Kähler manifolds, in the 1980's, Yau [56, p.139] initiated the program of approximating Kähler-Einstein metrics by embeddings into complex projective space by higher power sections of an ample holomorphic line bundle. The corresponding complete asymptotic expansion of Bergman kernels was established independently by Zelditch [57] and Catlin [9], which has important applications in the study of extremal metrics $[15,56]$. It 
can be viewed as the local version of the Hirzebruch-Riemann-Roch theorem. Thanks to the Kähler condition $d \omega=0$, the space of Weyl invariant polynomials on Kähler manifolds has a canonical basis represented by multi-digraphs. A closed formula has been discovered [51] for coefficients in the asymptotic expansion of the weighted Bergman kernel. We expect that a similar formula should exist for the heat kernel of the Laplacian operator on Kähler manifolds.

Karabegov and Schlichenmaier [35] showed that the logarithm of the diagonal value of the weighted Bergman kernel is the Karabegov classifying form of the Berezin quantization. There has been much work on deformation quantization of Kähler manifolds and its applications (see the recent survey [50]).

We summarize the definitions and properties of curvature tensors on Kähler manifolds. Let $(M, g)$ be a Kähler manifold of dimension $n$. Locally the Kähler form is given by $\omega_{g}=\frac{\sqrt{-1}}{2 \pi} \sum_{i, j=1}^{n} g_{i \bar{j}} d z_{i} \wedge d z_{\bar{j}}$. We will use the Einstein summation convention. The indices $i, j, k, \ldots$ run from 1 to $n$, while Greek indices $\alpha, \beta, \gamma$ may represent either $i$ or $\bar{i}$. Let $\operatorname{det} g$ be the determinant of the Hermitian matrix $\left(g_{i \bar{j}}\right)$ and $\left(g^{i \bar{j}}\right)$ be the inverse of the matrix $\left(g_{i \bar{j}}\right)$. We also use the notations

$$
\begin{gathered}
g_{j \bar{k} \alpha_{1} \alpha_{2} \ldots \alpha_{m}}:=\partial_{\alpha_{1} \alpha_{2} \ldots \alpha_{m}} g_{j \bar{k}}, \quad f_{\alpha_{1} \alpha_{2} \ldots \alpha_{m}}:=\partial_{\alpha_{1} \alpha_{2} \ldots \alpha_{m}} f \\
\left(R_{i \bar{j} k \bar{l} / \alpha_{1} \cdots \alpha_{m}}\right)_{\beta_{1} \cdots \beta_{r}}:=\partial_{\beta_{1} \cdots \beta_{r}} R_{i \bar{j} k \bar{l} / \alpha_{1} \cdots \alpha_{m}} .
\end{gathered}
$$

The curvature tensor is defined as

$$
R_{i \bar{j} k \bar{l}}=-g_{i \bar{j} k \bar{l}}+g^{m \bar{p}} g_{m \bar{j} \bar{l}} g_{i \bar{p} k} .
$$

The Ricci tensor is given by $R_{i \bar{j}}=g^{k \bar{l}} R_{i \bar{j} k \bar{l}}=-\partial_{i} \partial_{\bar{j}} \log (\operatorname{det} g)$ and the trace of the Ricci curvature is the scalar curvature $\rho=g^{i \bar{j}} R_{i \bar{j}}$.

The covariant derivative of a covariant tensor field $T_{\beta_{1} \ldots \beta_{p}}$ is defined by

$$
T_{\beta_{1} \ldots \beta_{p} / \gamma}=\partial_{\gamma} T_{\beta_{1} \ldots \beta_{p}}-\sum_{i=1}^{p} \Gamma_{\gamma \beta_{i}}^{\delta} T_{\beta_{1} \ldots \beta_{i-1} \delta \beta_{i+1} \ldots \beta_{p}},
$$

where the Christoffel symbols $\Gamma_{\beta \gamma}^{\alpha}=0$ except for $\Gamma_{j k}^{i}=g^{i \bar{l}} g_{j \bar{l} k}, \Gamma_{\bar{j} \bar{k}}^{\bar{i}}=g^{l \bar{i}} g_{l \bar{j} \bar{k}}$.

Lemma 1.1. On Kähler manifolds, we have $\partial_{i} g_{j \bar{k}}=\partial_{j} g_{i \bar{k}}, \partial_{\bar{l}} g_{j \bar{k}}=\partial_{\bar{k}} g_{j \bar{l}}$ and

$$
\begin{gathered}
\partial_{\alpha} g^{m \bar{l}}=-g^{p \bar{l}} g^{m \bar{q}} g_{p \bar{q} \alpha}, \quad \partial_{\alpha} \operatorname{det} g=\operatorname{det} g \cdot g^{m \bar{l}} g_{m \bar{l} \alpha} \\
R_{i \bar{j} k \bar{l}}=R_{i \bar{l} k \bar{j}}=R_{k \bar{j} i \bar{l}}(\text { first Bianchi), } \\
R_{i \bar{j} k \bar{l} / m}=R_{m \bar{j} k \bar{l} / i}=R_{i \bar{j} m \bar{l} / k}, \quad R_{i \bar{j} k \bar{l} / \bar{p}}=R_{i \bar{p} k \bar{l} / \bar{j}}=R_{i \bar{j} k \bar{p} / \bar{l}} \text { (second Bianchi) }
\end{gathered}
$$




$$
\begin{gathered}
T_{\beta_{1} \ldots \beta_{p} / i j}-T_{\beta_{1} \ldots \beta_{p} / j i}=0, \quad T_{\beta_{1} \ldots \beta_{p} / \bar{i} \bar{j}}-T_{\beta_{1} \ldots \beta_{p} / \bar{j} i}=0, \\
T_{\beta_{1} \ldots \beta_{p} / i \bar{j}}-T_{\beta_{1} \ldots \beta_{p} / \bar{j} i}=\sum_{k=1}^{p} R_{\beta_{k} i \bar{j}}^{\gamma} T_{\beta_{1} \ldots \beta_{k-1} \gamma \beta_{k+1} \ldots \beta_{p}} \text { (Ricci formula), }
\end{gathered}
$$

where $R_{\bar{l} \bar{j}}^{\bar{k}}=-g^{m \bar{k}} R_{m \bar{l} i \bar{j}}, R_{l i \bar{j}}^{k}=g^{k \bar{m}} R_{l \bar{m} i \bar{j}}$ and $R_{\bar{l} \bar{j}}^{k}=R_{l i \bar{j}}^{\bar{k}}=0$.

Recall that around each point $x$ on a Kähler manifold $M$, there exists a normal coordinate system such that $g_{i \bar{j}}(x)=\delta_{i j}, g_{i \bar{j} k_{1} \ldots k_{r}}(x)=g_{i \bar{j} \bar{l}_{1} \ldots \bar{l}_{r}}(x)=0$ for all $r \leq N \in \mathbb{N}$, where $N$ can be chosen arbitrary large.

Fix a normal coordinate system around $x$ on $M$. By (1) and (2), it is not difficult to see inductively that for any partial derivatives of metrics or curvature tensors, there exists a canonical tensor that coincides with it at $x$. We will use the operator $D$ to denote this correspondence. For example, $D\left(g_{i \bar{j} k \bar{l}}\right)=-R_{i \bar{j} k \bar{l}}$, $D\left(g_{i \bar{j} k \bar{l} \alpha}\right)=-R_{i \bar{j} k \bar{l} / \alpha}$ and

$$
D\left(\left(R_{i \bar{j} k \bar{l} / p_{1} \cdots p_{m}}\right)_{\bar{q}_{1} \cdots \bar{q}_{r}}\right)=R_{i \bar{j} k \bar{l} / p_{1} \cdots p_{m} \bar{q}_{1} \cdots \bar{q}_{r}} .
$$

As shown in [51, 52], tensor calculus on Kähler manifolds could be naturally formulated in terms of graphs. In particular, the Weyl invariants [22] can be represented by multi-digraphs. Each vertex in the graph is a partial derivatives of metrics in local coordinates. Note such graphs are not in one-to-one correspondence with tensorial vertices due to the Ricci formula.

In $\S 2$, we review Fefferman's program, especially the works of $[25,30]$. More detailed and nice expositions can be found in [29]. In $\S 3$, building on work of Engliš [17], we prove a graph-theoretic formula for Nakazawa's asymptotic expansion. In $\S 4-\S 6$, we will prove closed formulae for the above operator $D$ as a summation over rooted trees with external legs, by canonically associate a tensor expression to each rooted tree. As an illustration of the efficiency of these formulae, we rederive the weight 3 coefficient in the asymptotic expansion of the weighted Bergman kernel.

Acknowledgements The author thanks Professors Kefeng Liu and Shing-Tung Yau for helpful conversations and encouragements. The author also thanks Professors Miroslav Engliš and Kengo Hirachi for helpful communications, whose influence on this paper is evident. 


\section{Fefferman's PRogram and CR invariants: A Review}

Let $\Omega$ be a bounded strictly pseudoconvex domain in $\mathbb{C}^{n}$ with smooth boundary. If $r \in C^{\infty}(\bar{\Omega})$ is a defining function in the sense $\Omega=\{r>0\}$ with $d r \neq 0$ on $\partial \Omega$, then by the pioneering work of Fefferman [20] (see also [8]), the boundary singularity of the Bergman kernel has the form

$$
K(z)=\frac{n !}{\pi^{n}}\left(\frac{\varphi(z)}{r(z)^{n+1}}+\psi(z) \log r(z)\right), \quad \varphi, \psi \in C^{\infty}(\bar{\Omega}) .
$$

A weighted analogue of Fefferman's expansion was obtained by Engliš [18].

Hörmander [32] proved that $r(z)^{n+1} K(z) \rightarrow \frac{n !}{\pi^{n}} J[r]\left(z_{0}\right)$ as $z \rightarrow z_{0} \in \partial \Omega$, where $J[r]$ denotes the complex Monge-Ampère operator defined by

$$
J[r]=(-1)^{n} \operatorname{det}\left(\begin{array}{cc}
r & \partial r / \partial \bar{z}_{j} \\
\partial r / \partial z_{i} \partial^{2} r / \partial z_{i} \partial \bar{z}_{j}
\end{array}\right)_{1 \leq i, j \leq n} .
$$

By (9), we have $\varphi=J[r]$ on $\partial \Omega$. Starting from an arbitrary smooth defining function of $\Omega$, Fefferman [21] devised a recursive algorithm to explicitly construct another defining function $r^{F} \in C^{\infty}(\bar{\Omega})$ satisfying

$$
J\left[r^{F}\right]=1+O^{n+1}\left(r^{F}\right), \quad r^{F}>0 \text { in } \Omega,\left.\quad r^{F}\right|_{\partial \Omega}=0,
$$

where $O^{n+1}\left(r^{F}\right)$ denotes a term of the form $\left(r^{F}\right)^{n+1} f$ with $f \in C^{\infty}(\bar{\Omega})$.

Let $r^{F}$ be a Fefferman's defining function of $\Omega$. Define a Lorentz-Kähler metric

$$
g=\sum_{0 \leq i, j \leq n} \frac{\partial^{2} r_{\#}}{\partial z_{i} \partial \bar{z}_{j}} d z_{i} d \bar{z}_{j}, \quad r_{\#}\left(z_{0}, z\right)=\left|z_{0}\right|^{2} r^{F}(z) \text { on } \mathbb{C}^{*} \times \bar{\Omega},
$$

called Fefferman's ambient metric associated with $\partial \Omega$. From the curvature tensor $R$ of $g$, Fefferman [22] constructed Weyl invariants by complete contractions of covariant derivatives $R^{(p, q)}:=R_{a_{1} \bar{b}_{1} a_{2} \bar{b}_{2} / a_{3} \cdots a_{p} \bar{b}_{3} \cdots \bar{b}_{q}}$, e.g. the following Weyl invariant

$$
W_{\#}=\operatorname{contr}\left(R^{\left(p_{1}, q_{1}\right)} \otimes \cdots \otimes R^{\left(p_{s}, q_{s}\right)}\right),
$$

is defined to be of weight $\sum_{j=1}^{s}\left(p_{j}+q_{j}\right) / 2-s$ and gives rise to a function $W=$ $\left.W_{\#}\right|_{z_{0}=1}$ on $\bar{\Omega}$. Fefferman proposed a program [22] to express $\varphi, \psi$ in (9) as linear combinations of Weyl invariants $W_{k}$ of weight $k$ such that

$$
\varphi=\sum_{k=0}^{n} W_{k} r^{k}+O^{n+1}(r), \quad \psi=\sum_{k=0}^{\infty} W_{k+n+1} r^{k}+O^{\infty}(r),
$$


where $O^{\infty}(r)$ means that $\psi=\sum_{k=0}^{m} W_{k+n+1}[r] r^{k}+O^{m+1}(r)$ for any $m \geq 0$. The expansion of $\varphi$ was proved by Fefferman [22] and Bailey et al. [3] for any Fefferman's defining function $r=r^{F}$. The expansion of $\psi$ was proved by Hirachi [27] for more refined defining functions than (11). Both are very deep works.

According to Fefferman [22], the restriction of $W_{k}$ to $\partial \Omega$ gives a CR invariant of weight $k$. CR invariants for strictly pseudoconvex hypersurfaces can be more conveniently defined using Moser's normal form in analogy to the normal coordinate system in Riemannian geometry. Let $\left(z^{\prime}, z_{n}\right)=\left(z_{1}, \ldots, z_{n}\right) \in \mathbb{C}^{n}$. A hypersurface $0 \in \partial \Omega \subset \mathbb{C}^{n}$ with local equation

$$
2 u=\left|z^{\prime}\right|^{2}+\sum_{|\alpha|,|\beta| \geq 2, k \geq 0} A_{\alpha \bar{\beta}}^{k}(v) v^{k} z_{\alpha}^{\prime} \bar{z}_{\beta}^{\prime}, \quad z_{n}=u+i v
$$

is said to be in Moser's normal form if the coefficients $A_{\alpha \bar{\beta}}^{k}$ satisfy: (i) $A_{\alpha \bar{\beta}}^{k}=\overline{A_{\beta \bar{\alpha}}^{k}}$;

(ii) $\operatorname{tr}\left(A_{2 \overline{2}}\right)=0$, i.e. $\sum_{p=1}^{n-1} A_{p i \bar{p} \bar{j}}^{k}=0$ for all $k, i, j$;

(iii) $\operatorname{tr}\left(A_{2 \overline{3}}\right)=0$, i.e. $\sum_{p, q=1}^{n-1} A_{p q \bar{p} \bar{q} \bar{j}}^{k}=0$ for all $k, j$;

(iv) $\operatorname{tr}\left(A_{3 \overline{3}}\right)=0$, i.e. $\sum_{p, q, r=1}^{n-1} A_{p q r \bar{p} \bar{q} \bar{r}}^{k}=0$ for all $k$.

A classical result of Chern and Moser [12] says that any real analytic hypersurface may be placed in Moser's normal form through a biholomorphic map.

Definition 2.1 ([22, 25, 30]). Denote by $N\left(A_{\alpha \bar{\beta}}^{k}\right)$ a real hypersurface in normal form (14). A polynomial $P$ in variables $A_{\alpha \bar{\beta}}^{k}$ is said to be a CR invariant of weight $w \in \mathbb{N}_{\geq 0}$ if it satisfies the transformation law $P\left(A_{\alpha \bar{\beta}}^{k}\right)=$ $\left|\operatorname{det} \Phi^{\prime}(0)\right|^{2 w /(n+1)} P\left(B_{\alpha \bar{\beta}}^{k}\right)$ for any biholomorphic mapping $\Phi: N\left(A_{\alpha \bar{\beta}}^{k}\right) \rightarrow N\left(B_{\alpha \bar{\beta}}^{k}\right)$ preserving the origin.

Let $I_{w}$ denote the set of $\mathrm{CR}$ invariants of weight $w$. Then every $P \in I_{w}$ is a homogeneous polynomial of weight $w$ if we define the weight of $A_{\alpha \bar{\beta}}^{k}$ to be $(|\alpha|+|\beta|) / 2+k-1$. Graham $[25]$ proved the following:

Theorem $2.2([25])$. (i) Let $n=2$. Then $I_{1}=I_{2}=\{0\}$ and $\operatorname{dim} I_{3}=\operatorname{dim} I_{4}=1$. Moreover, $I_{3}$ and $I_{4}$ are respectively spanned by $A_{4 \overline{4}}^{0}$ and $\left|A_{2 \overline{4}}^{0}\right|^{2}$.

(ii) Let $n \geq 3$. Then $I_{1}=\{0\}$ and $\operatorname{dim} I_{2}=1$. Moreover, $I_{2}$ is spanned by $\left\|A_{2 \overline{2}}^{0}\right\|^{2}=\sum\left|A_{\alpha \bar{\beta}}^{0}\right|^{2}$, where the summation runs over $|\alpha|=|\beta|=2$. 
When $n=2$, a basis of $\operatorname{dim} I_{5}=2$ has been determined in $[25,30]$ and a basis of $\operatorname{dim} I_{6}=3$ has been determined by Hirachi [28].

For the Dirichlet problem of the complex Monge-Ampère equation

$$
J[u]=1, \quad u>0 \text { in } \Omega,\left.\quad u\right|_{\partial \Omega}=0,
$$

Cheng-Yau [11] proved that there exists a unique solution $u \in C^{\infty}(\Omega) \cap C^{n+3 / 2-\epsilon}(\bar{\Omega})$ for any $\epsilon>0$. Lee-Melrose [37] proved that for any smooth defining function $r$, Cheng-Yau's solution has an asymptotic expansion

$$
u \sim r \sum_{k=0}^{\infty} \eta_{k} \cdot\left(r^{n+1} \log r\right)^{k}, \quad \eta_{k} \in C^{\infty}(\bar{\Omega}),
$$

which implies that $u \in C^{n+2-\epsilon}(\bar{\Omega})$ for any $\epsilon>0$ improving Cheng-Yau's estimate. However, the solution to (15) is not $C^{\infty}$ smooth up to the boundary, so we have to use Fefferman's defining function $r^{F}$ when studying the invariant expansions (13).

Let us fix $r=r^{\mathrm{F}}$ and $a \in C^{\infty}(\partial \Omega)$ locally near $0 \in \partial \Omega$. Then Graham [26] proved that there exists a unique formal series $u$ of the form (16) satisfying

$$
J[u]=1+O\left(r^{\infty}\right), \quad \eta_{0}=1+a r^{n+1}+O\left(r^{n+2}\right)
$$

near $0 \in \partial \Omega$. For any $k \geq 1, \eta_{k}$ modulo $O\left(r^{n+1}\right)$ is independent of $r=r^{\mathrm{F}}$ and $a$. Each $\left.\eta_{k}\right|_{\partial \Omega}$ modulo $O\left(r^{n+1}\right)$ is a CR invariant of weight $k(n+1)$. From Theorem 2.2, Graham [25] proved that:

Theorem $2.3([25])$. (i) Let $n=2$. Then $\eta_{1}=4 A_{4 \overline{4}}^{0}$ and the singularity of the Bergman kernel (9) has the expansions

$$
\varphi=1+O\left(r^{3}\right), \quad \psi=-3 \eta_{1}+c\left|A_{2 \overline{4}}^{0}\right|^{2} r+O\left(r^{2}\right),
$$

where $c$ is a constant independent on $\Omega$.

(ii) Let $n \geq 3$. There is a constant $c_{n}$ depending only on $n$ such that

$$
\varphi=1+c_{n}\left\|A_{2 \overline{2}}^{0}\right\|^{2} r^{2}+O\left(r^{3}\right)
$$

Hirachi, Komatsu and Nakazawa [30] gave two different methods of identifying the above universal constants and proved similar expansions for the Szegö kernel. In [31], they extended the expansion of $\psi$ in dimension 2 to weight 5 . We outline their proof using Nakazawa's explicit asymptotic expansion for Reinhardt domains. 
Theorem 2.4 ([30]). The constants in (18) and (19) are given by $c=24 / 5$ and $n(n-1) c_{n}=2 / 3$.

In the rest of the section, we assume $\Omega \subset \mathbb{C}^{n}$ is a bounded strictly pseudoconvex complete Reinhardt domain. Its logarithmic real representation domain is given by

$$
-\log |\Omega|=\left\{(x, y) \in \mathbb{R}^{n-1} \times \mathbb{R} \mid\left(e^{-x_{1}}, \ldots, e^{-x_{n-1}}, e^{-y}\right) \in \Omega\right\} .
$$

First we assume $n=2$. Let $f(x):=\inf \{y \in \mathbb{R}|(x, y) \in-\log | \Omega \mid\}$. Then $\lambda=y-f(x)(>0)$ is a defining function of $\partial \Omega \cap\left\{z_{1} z_{2} \neq 0\right\}$. We make change of variables $(x, y) \rightarrow(\lambda, v)$ with $v=f^{\prime}(x)$ and set $p(v)=f^{\prime \prime}(x)$, the hodograph transformation. The following asymptotic expansion in dimension 2 was proved by Nakazawa [48] improving work of Boichu and Coeuré [7].

Theorem 2.5 ([48, 30]). Let $n=2$. Near $\partial \Omega \cap\left\{z_{1} z_{2} \neq 0\right\}$, we have

$$
K(z)=\frac{2}{\pi^{2}} J[\lambda]\left(\frac{\widetilde{\varphi}(v, \lambda)}{\lambda^{3}}+\widetilde{\psi}(v, \lambda) \log \lambda\right),
$$

where $J[\lambda]=\frac{p}{\left|4 z_{1} z_{2}\right|^{2}}$. Let $e_{1}=p^{\prime \prime}, e_{2}=\left(p p^{(3)}\right)^{\prime}, e_{3}=\left(p^{2} p^{(4)}\right)^{\prime \prime}, e_{41}=e_{1} e_{3}$, $e_{42}=\left(p e_{3}^{\prime}\right)^{\prime}$ and $e_{43}=\left(p p^{(4)}\right)^{2}$. Then

$$
\widetilde{\varphi}(v, \lambda)=1+\frac{\lambda}{4} e_{1}+\frac{\lambda^{2}}{12} e_{2}, \quad \widetilde{\psi}(v, \lambda)=\frac{-e_{3}}{48}+\frac{\lambda}{480}\left(2 e_{42}+e_{43}-e_{41}\right)+O\left(\lambda^{2}\right) .
$$

Theorem 2.5 and the following lemma immediately implies $c=24 / 5$ in (18).

Lemma $2.6([30])$. Under the notation of the above theorem, we have $\left|A_{2 \overline{4}}^{0}\right|^{2}=$ $J[\lambda]^{4 / 3} e_{43} / 48^{2}, r^{F}=J[\lambda]^{-1 / 3}\left(\widetilde{r}+O\left(\lambda^{4}\right)\right)$ and $\eta_{1}=J[\lambda]\left(\widetilde{\eta}_{1}+O\left(\lambda^{2}\right)\right)$, where

$$
\widetilde{r}=\lambda-\frac{\lambda^{2}}{12} e_{1}-\frac{\lambda^{3}}{36}\left(e_{2}-\frac{e_{1}^{2}}{2}\right), \quad \widetilde{\eta}_{1}=\frac{e_{3}}{144}-\frac{\lambda}{720}\left(e_{42}-\frac{e_{41}}{2}\right) .
$$

Next we assume $n \geq 3$. Let $\Omega \subset \mathbb{C}^{n}$ be a bounded strictly pseudoconvex complete Reinhardt domain satisfying $-\log |\Omega|=\left\{\lambda:=y-\left(f_{1}(x)+\cdots+f_{n-1}(x)\right)>\right.$ $0\}$ with hodograph variables $v_{j}=f_{j}^{\prime}\left(x_{j}\right)$ and $p_{j}\left(v_{j}\right)=f_{j}^{\prime \prime}\left(x_{j}\right)$. We introduce

$$
e_{1}=\sum_{j=1}^{n-1} p_{j}^{\prime \prime}, \quad e_{21}=\sum_{j=1}^{n-1}\left(p_{j} p_{j}^{\prime \prime \prime}\right)^{\prime}, \quad e_{22}=\sum_{j=1}^{n-1}\left(p_{j}^{\prime \prime}\right)^{2}, \quad e_{23}=\sum_{j \neq k} p_{j}^{\prime \prime} p_{k}^{\prime \prime} .
$$

Theorem 2.7 ([30]). Under the above notation, we have

$$
\left\|A_{2 \overline{2}}^{0}\right\|^{2}=\frac{J[\lambda]^{2 /(n+1)}}{16 n(n+1)}\left((n-2)(n-1) e_{22}+2 e_{23}\right),
$$


$r^{F}=J[\lambda]^{\frac{-1}{n+1}}\left(\lambda-\frac{e_{1} \lambda^{2}}{2 n(n+1)}+\frac{-n(n+1) e_{21}+\left(n^{2}-1\right) e_{22}-e_{23}}{6(n-1) n^{2}(n+1)^{2}} \lambda^{3}+O\left(\lambda^{4}\right)\right)$.

The Bergman kernel has the expansion

$$
K(z)=\frac{n !}{\pi^{n}} J[\lambda]\left(\frac{\widetilde{\varphi}(v, \lambda)}{\lambda^{n+1}}+\widetilde{\psi}(v, \lambda) \log \lambda\right),
$$

where $J[\lambda]=\frac{p}{4^{n}\left|z_{1} \cdots z_{n}\right|^{2}}$ and

$$
\widetilde{\varphi}(v, \lambda)=1+\frac{\lambda}{2 n} e_{1}+\frac{\lambda^{2}}{n(n-1)}\left(\frac{1}{6} e_{21}+\frac{1}{8} e_{23}\right)+O\left(\lambda^{3}\right) .
$$

Theorem 2.7 immediately implies $c_{n}=\frac{2}{3 n(n-1)}$ in (19). Explicit graph theoretic formulae for the coefficients of (20) and (21) will be proved in $\S 3$.

\section{The asymptotic expansion of Bergman kernels}

There has been much interest in explicit formulae of Fefferman's asymptotic expansions for (unweighted) Bergman kernels and Szegö kernels (see [4, 34, 36, 48]). In [17], Engliš studied the asymptotic expansion of a Laplace integral and proved a recursion relation for its coefficients. As an application, he derived a remarkable formula (Theorem 3.8) of Fefferman's invariants for the (unweighted) Bergman kernel of strictly pseudoconvex Hartogs domains using the Forelli-Rudin construction. We will show that some key quantities in Engliš' formula can be expressed as explicit summations over strongly connected graphs and using an observation of Nakazawa (Lemma 3.9), Engliš' formula can be drastically simplified when the domain is complete Reinhardt. The resulting graph-theoretic formula (Theorem 3.10) is also more computationally efficient. Similar formula also exists for Szegö kernels [19].

Theorem 3.1 (Engliš [17]). Let $\Omega$ be a strongly pseudoconvex domain in $\mathbb{C}^{n}$ with real analytic boundary. Then there is an asymptotic expansion for the Laplace integral as $\alpha \rightarrow \infty$,

$$
\int_{\Omega} f(y) e^{-\alpha(\Phi(x)+\Phi(y)-\Phi(x, y)-\Phi(y, x))} \frac{|\operatorname{det} g(x, y)|^{2}}{\operatorname{det} g(x)} d y \sim \pi^{n} \sum_{j \geq 0} \alpha^{-n-j} \mathcal{R}_{j}(f)(x),
$$

where $\Phi(x, y)$ and $\operatorname{det} g(x, y)$ are the almost analytic extensions of the Kähler potential $\Phi(x)$ and $\operatorname{det} g(x)$ respectively, and $\mathcal{R}_{j}: C^{\infty}(\Omega) \rightarrow C^{\infty}(\Omega)$ are explicit 
differential operators defined by

$$
\mathcal{R}_{j} f(x)=\left.\frac{1}{(\operatorname{det} g)^{2}} \sum_{k=j}^{3 j} \frac{1}{k !(k-j) !} L^{k}\left[f(y)|\operatorname{det} g(x, y)|^{2} S(x, y)^{k-j}\right]\right|_{y=x},
$$

where $L$ is the (constant-coefficient) differential operator

$$
L f(y)=g^{i \bar{j}}(x) \partial_{i} \partial_{\bar{j}} f(y)
$$

and the function $S(x, y)$ satisfies

$$
\begin{gathered}
S=\partial_{\alpha} S=\partial_{\alpha \beta} S=\partial_{i_{1} i_{2} \ldots i_{m}} S=\partial_{\bar{i}_{1} \bar{i}_{2} \ldots \bar{i}_{m}} S=0 \quad \text { at } y=x, \\
\left.\partial_{i \bar{j} \alpha_{1} \alpha_{2} \ldots \alpha_{m}} S\right|_{y=x}=-\partial_{\alpha_{1} \alpha_{2} \ldots \alpha_{m}} g_{i \bar{j}}(x), \quad m \geq 1 .
\end{gathered}
$$

Denote by $\mathcal{K}_{\alpha}(x, y)$ the reproducing kernel of the weighted Bergman space of all holomorphic functions on $\Omega$ square-integrable with respect to the measure $e^{-\alpha \Phi} d x$. According to Engliš [17], $\mathcal{K}_{\alpha}(x, y)$ has an asymptotic expansion in a small neighborhood of the diagonal as $\alpha \rightarrow \infty$,

$$
\mathcal{K}_{\alpha}(x, y)=e^{\alpha \Phi(x, y)} \operatorname{det} g(x, y) \frac{1}{\pi^{n}} \sum_{k=0}^{\infty} \mathcal{B}_{k}(x, y) \alpha^{n-k} .
$$

The corresponding Berezin transform is given by

$$
\mathcal{I}_{\alpha} f(x)=\int_{\Omega} f(y) \frac{\left|\mathcal{K}_{\alpha}(x, y)\right|^{2}}{\mathcal{K}_{\alpha}(x, x)} e^{-\alpha \Phi(y)} d y,
$$

which has an asymptotic expansion as $\alpha \rightarrow \infty$,

$$
\mathcal{I}_{\alpha} f(x)=\sum_{k=0}^{\infty} \mathcal{Q}_{k} f(x) \alpha^{-k}
$$

The following lemma is the key result we will use, which slightly refines the formulae of Engliš [17].

Lemma 3.2. We have $\mathcal{Q}_{0}=i d$ and $\mathcal{B}_{0}=1$. For $k \geq 1$,

$$
\begin{gathered}
\mathcal{Q}_{k} f(x)=\left.\sum_{j=0}^{k} \sum_{i=0}^{k-j} \mathcal{R}_{j}\left(\mathcal{B}_{i}(x, y) \mathcal{B}_{k-j-i}(y, x) f(y)\right)\right|_{y=x}-\sum_{m=1}^{k} \mathcal{B}_{m}(x) \mathcal{Q}_{k-m} f(x), \\
\mathcal{B}_{k}(x)=-\sum_{\substack{i+j=k \\
i, j \geq 1}} \mathcal{B}_{i}(x) \mathcal{B}_{j}(x)-\left.\sum_{\substack{\ell+i+j=k \\
1 \leq \ell \leq k}} \mathcal{R}_{\ell}\left(\mathcal{B}_{i}(x, y) \mathcal{B}_{j}(y, x)\right)\right|_{y=x} .
\end{gathered}
$$


Proof. By multiplying $\mathcal{K}_{\alpha}(x, x)$ to both sides of (25) and using (24) and (26), we get

$$
\begin{aligned}
\sum_{m=0}^{\infty} \mathcal{B}_{m}(x, y) \sum_{i=0}^{\infty} \mathcal{Q}_{i} f(x) & \alpha^{n-m-i}=\frac{1}{\pi^{n}} \int_{\Omega} f(y) e^{-\alpha(\Phi(x)+\Phi(y)-\Phi(x, y)-\Phi(y, x))} \\
\times & \frac{|\operatorname{det} g(x, y)|^{2}}{\operatorname{det} g(x)} \sum_{i, m=0}^{\infty} \mathcal{B}_{i}(x, y) \mathcal{B}_{m}(y, x) \alpha^{2 n-m-i} d y
\end{aligned}
$$

By applying Theorem 3.1 to the right-hand side of the above equation and equating the coefficients of $\alpha^{n-k}$, we get (27).

Since $Q_{0}=i d$ and $Q_{k}(f)=0$ when $k \geq 1$ and $f$ is either holomorphic or antiholomorphic, by substituting $f=1$ in (27), we get (28).

As noticed by Engliš [17], in a normal coordinate system around $x$, the operators $\mathcal{R}_{j}$ in (23) simplify to

$$
\mathcal{R}_{j} f(x)=\left.\sum_{k=j}^{2 j} \frac{1}{k !(k-j) !} L^{k}\left(f S^{k-j}\right)\right|_{y=x} .
$$

Before proceeding we need to introduce parallel notions for graphs and pointed graphs representing Weyl invariant polynomials in jets of metrics and functions.

A digraph or simply a graph $G=(V, E)$ is defined to be a finite directed multigraph which may have multi-edges and loops. A vertex $v$ of a digraph $G$ is called stable if $\operatorname{deg}^{-}(v) \geq 2 \operatorname{deg}^{+}(v) \geq 2$, i.e. both the inward and outward degrees of $v$ are no less than 2. A vertex $v$ is called semistable if we have

$$
\operatorname{deg}^{-}(v) \geq 1, \operatorname{deg}^{+}(v) \geq 1 \operatorname{deg}^{-}(v)+\operatorname{deg}^{+}(v) \geq 3 .
$$

The weight of a digraph $G$ is defined to be the integer $w(G)=|E|-|V|$. A digraph $G$ is stable (semistable) if each vertex of $G$ is stable (semistable). The set of semistable and stable graphs of weight $k$ will be denoted by $\mathcal{G}^{s s}(k)$ and $\mathcal{G}(k)$ respectively. A directed edge $u v$ of a semistable digraph is called contractible if $u \neq v$ and at least one of the following two conditions holds: (i) $\operatorname{deg}^{+}(u)=1$; (ii) $\operatorname{deg}^{-}(v)=1$. A semistable graph $G$ is called stabilizable if after contractions of a finite number of contractible edges of $G$, the resulting graph becomes stable, which is called the stabilization graph of $G$ and denoted by $G^{s}$. 
A (one-)pointed graph $\Gamma=(V \cup\{\bullet\}, E)$ is defined to be a digraph with a distinguished vertex labeled by $f . G$ or $\Gamma$ is called semistable (stable) if each ordinary vertex $v \in V$ is semistable (stable). The weight of a pointed graph $\Gamma=(V \cup\{\bullet\}, E)$ is defined to be $w(\Gamma)=|E|-|V|$. By abuse of notation, we denote $V(\Gamma)=V \cup\{\bullet\}$. The set of semistable and stable pointed graphs of weight $k$ will be denoted by $\mathcal{G}_{1}^{s s}(k)$ and $\mathcal{G}_{1}(k)$ respectively. Denote by $\operatorname{Aut}(\Gamma)$ the set of all automorphisms of the pointed graph $\Gamma$ fixing the distinguished vertex. A directed edge $u v$ of a semistable pointed graph is called contractible if $u \neq v$ and at least one of the following two conditions holds: (i) $u \in V$ and $\operatorname{deg}^{+}(u)=1$; (ii) $v \in V$ and $\operatorname{deg}^{-}(v)=1$. A semistable pointed graph $\Gamma$ is called stabilizable if after contractions of a finite number of contractible edges of $\Gamma$, the resulting graph becomes stable, which is called the stabilization graph of $\Gamma$ and denoted by $\Gamma^{s}$.

We can canonically associate a polynomial in the variables $\left\{g_{i \bar{j} \alpha}\right\}_{|\alpha| \geq 1}$ or $\left\{f_{\alpha}\right\}_{|\alpha| \geq 0}$ to a semistable graph or pointed graph, such that each ordinary vertex represents a partial derivative of $g_{i \bar{j}}$, the distinguished vertex represents a partial derivative of $f$ and each edge represents the contraction of a pair of indices.

Remark 3.3. Note that the definition of stablizable semistable (pointed) graphs is independent of the order of edge-contractions (cf. [53, Lem. 3.1]). Engliš [17] showed that $\mathcal{B}_{k}, \mathcal{R}_{k}, \mathcal{Q}_{k}$ are Weyl invariant polynomials in the variables $\left\{g_{i \bar{j} \alpha}\right\}$ or $\left\{f_{\alpha}\right\}$, i.e. invariant under transformation of coordinates. Assume that the following two Weyl invariant polynomials are expressed, in a normal coordinate system, as summations over stable graphs and pointed graphs respectively,

$$
P_{1}=\sum_{G \in \mathcal{G}(k)} c(G) G \text { and } P_{2}=\sum_{\Gamma \in \mathcal{G}_{1}(k)} c(\Gamma) \Gamma .
$$

The main result of [53] shows that they may be invariantly expressed as summations over stabilizable semistable (pointed) graphs,

$$
\begin{aligned}
P_{1} & =\sum_{G \in \mathcal{G}^{s s}(k)}^{\text {stabilizable }} \frac{(-1)^{|V(G)|-\left|V\left(G^{s}\right)\right|}\left|\operatorname{Aut}\left(\mathrm{G}^{\mathrm{s}}\right)\right| c\left(G^{s}\right)}{|\operatorname{Aut}(\mathrm{G})|} G, \\
P_{2} & =\sum_{\Gamma \in \mathcal{G}_{1}^{\text {ss }}(k)}^{\text {stabilizable }} \frac{(-1)^{|V(\Gamma)|-\left|V\left(\Gamma^{s}\right)\right|}\left|\operatorname{Aut}\left(\Gamma^{\mathrm{s}}\right)\right| c\left(\Gamma^{s}\right)}{|\operatorname{Aut}(\Gamma)|} \Gamma,
\end{aligned}
$$

which are valid in arbitrary holomorphic coordinates. 
In the following, we use the notations

$$
\mathcal{B}_{k}=\sum_{G \in \mathcal{G}(k)} \mathcal{B}_{G} G, \quad \mathcal{R}_{k} f=\sum_{\Gamma \in \mathcal{G}_{1}(k)} \mathcal{R}_{\Gamma} \Gamma, \quad \mathcal{Q}_{k} f=\sum_{\Gamma \in \mathcal{G}_{1}(k)} \mathcal{Q}_{\Gamma} \Gamma
$$

Lemma 3.4. Let $\Gamma=(V \cup\{\bullet\}, E) \in \mathcal{G}_{1}$ be a stable pointed graph. Then

$$
\mathcal{R}_{\Gamma}=\frac{(-1)^{|V(\Gamma)|+1}}{|\operatorname{Aut}(\Gamma)|} .
$$

Proof. It follows from (30) if we regard $L^{k}$ as $k$ edges, $S^{k-j}$ as $k-j$ vertices and $k !(k-j)$ ! the symmetry factor.

Corollary 3.5. In any holomorphic coordinates, we have

$$
\mathcal{R}_{k} f=\sum_{\Gamma \in \mathcal{G}_{1}^{s s}(k)}^{\text {stabilizable }} \frac{(-1)^{|V(\Gamma)|+1}}{|\operatorname{Aut}(\Gamma)|} \Gamma .
$$

Proof. It follows from (33).

In the following, we call a graph strong if it is strongly connected. We call a graph quasi-strong if all of its connected components are strong. A strongly connected component (SCC) of a digraph $G$ is called a source (sink) if it has only outward (inward) edges in $G$. A connected graph is strong if and only it has no proper source or sink.

Theorem 3.6. Let $G \in \mathcal{G}$ and $\Gamma \in \mathcal{G}_{1}$. Then

$$
\begin{aligned}
& \mathcal{B}_{G}=\left\{\begin{array}{lr}
\frac{(-1)^{|V(G)|+n(G)}}{|\operatorname{Aut}(G)|} & \text { if } G \text { is quasi-strong with } n(G) \text { components, } \\
0 & \text { otherwise. }
\end{array}\right. \\
& \mathcal{Q}_{\Gamma}= \begin{cases}\frac{(-1)^{|V(\Gamma)|+1}}{|\operatorname{Aut}(\Gamma)|} & \text { if } \Gamma \text { is strong, } \\
0 & \text { otherwise. }\end{cases}
\end{aligned}
$$

Proof. First assume that $G$ is strongly connected. Let us look at the right-hand side of $(28)$. The first term $\mathcal{B}_{i}(x) \mathcal{B}_{j}(x)$ only contributes disconnected graphs. In the second term $\left.R_{\ell}\left(\mathcal{B}_{i}(x, y) \mathcal{B}_{j}(y, x)\right)\right|_{y=x}$, the two factors $\mathcal{B}_{i}(x, y)$ and $\mathcal{B}_{j}(y, x)$ are sink and source respectively. Since $G$ is strong, we must have $i=j=0$. So 
it is not difficult to see from (28) and (35) that

$$
\mathcal{B}_{G}=-\mathcal{R}_{G \amalg\{\bullet\}}=\frac{(-1)^{|V(G)|+1}}{|\operatorname{Aut}(G)|},
$$

where $G \amalg\{\bullet\}$ is the disjoint union of $G$ and the distinguished vertex $\bullet$.

Denote $\underline{n}=\{1, \cdots, n\}$. If $G=G_{1} \amalg \cdots \coprod G_{n}$ is disjoint union of strongly connected subgraphs, by inducting on $w(G)$ and using (28), we have

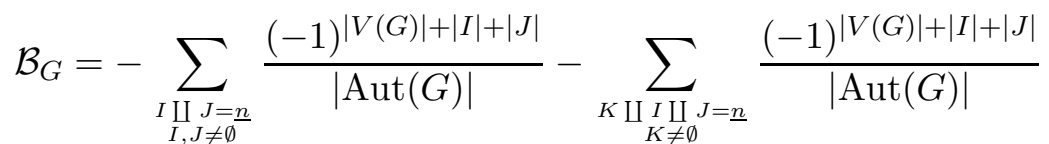

$$
\begin{aligned}
& =-\frac{(-2)^{|V(G)|+n}-2(-1)^{|V(G)|+n}}{|\operatorname{Aut}(G)|}-\frac{(1-1-1)^{|V(G)|+n}-(-2)^{|V(G)|+n}}{|\operatorname{Aut}(G)|} \\
& =\frac{(-1)^{|V(G)|+n}}{|\operatorname{Aut}(G)|} \text {. }
\end{aligned}
$$

If some $G_{i}$ in $G=G_{1} \amalg \cdots \coprod G_{n}$ is not strongly connected, then $G_{i}$ has a proper sink $C$. In order to prove $\mathcal{B}_{G}=0$, we note that in $\left.R_{\ell}\left(\mathcal{B}_{i}(x, y) \mathcal{B}_{j}(y, x)\right)\right|_{y=x}$, the sink $C$ may either belong to $B_{i}(x, y)$ or $R_{\ell}$, actually the contributions of these two cases to $G$ exactly cancel out. The argument is similar to the proof of $[52$, Prop. 3.3]. We omit the details. So we conclude the proof of the formula (37).

The formula for $\mathcal{Q}_{\Gamma}$ follows from (27), (35) and (37) by using the same argument as [52, Thm. 3.4].

Corollary 3.7. In any holomorphic coordinates, we have

$$
\begin{aligned}
\mathcal{B}_{k} & =\sum_{G \in \mathcal{G}^{s s}(k)}^{\text {quasi-strong }} \frac{(-1)^{|V(G)|+n(G)}}{|\operatorname{Aut}(G)|} G, \\
\mathcal{Q}_{k} f & =\sum_{\Gamma \in \mathcal{G}_{1}^{\text {ss }}(k)}^{\text {strong }} \frac{(-1)^{|V(\Gamma)|+1}}{|\operatorname{Aut}(\Gamma)|} \Gamma .
\end{aligned}
$$

Proof. In [53, Lem. $3.4 \&$ Lem. 4.5], we proved that the stabilization graph of a semistable (pointed) graph $G$ is strong if and only if $G$ is strong. So the corollary follows from (32) and (33). 
It is not difficult to see that (41) is equivalent to

$$
\sum_{k=0}^{\infty} \mathcal{B}_{k} \nu^{k}=\exp \left(\sum_{k=1}^{\infty} \sum_{G \in \mathcal{G}^{s s}(k)}^{\text {strong }} \nu^{k} \frac{(-1)^{|V(G)|+1}}{|\operatorname{Aut}(G)|} G\right)
$$

where in the right-hand side, $G$ runs over only strong graphs.

Theorem 3.8 (Engliš [17]). Let $\Omega$ be a strongly pseudoconvex domain in $\mathbb{C}^{N}$ with real-analytic boundary, $\Phi$ a strictly plurisubharmonic real-analytic defining function for $\Omega, g_{i \bar{j}}$ the Kähler metric defined by the potential $\Phi, d$ a positive integer, and $\widetilde{K}(z, w)$ the (ordinary unweighted) Bergman kernel of the Hartogs domain

$$
\widetilde{\Omega}=\left\{z=\left(z_{1}, z_{2}\right) \in \Omega \times \mathbb{C}^{d}:\left\|z_{2}\right\|^{2}<e^{-\Phi\left(z_{1}\right)}\right\} .
$$

Then (i) as z approaches a point of $\partial \widetilde{\Omega} \backslash\left\{z_{2}=0\right\}$, the reproducing kernel $\widetilde{K}(z, z)$. admits an asymptotic expansion

$$
\widetilde{K}(z, z)=\sum_{l=0}^{\infty} c_{l}\left(z_{1}\right) \cdot u_{d+N-l}\left(\left\|z_{2}\right\|^{2} e^{\Phi\left(z_{1}\right)}\right),
$$

in the sense that the partial sum of the first $l$ terms of the right-hand side differs from the left-hand side by a function which is $O\left(u_{d+N-l}\left(\left\|z_{2}\right\|^{2} e^{\Phi\left(z_{1}\right)}\right)\right)$ if $l \leqq$ $d+N+1$, and is in $C^{l-(d+N+2)}\left(\widetilde{\Omega} \backslash\left\{z_{2}=0\right\}\right)$ if $l \geqq d+N+2$. Here the function $u_{l}(w)$ is given by

$$
\begin{aligned}
u_{l}(w) & =\sum_{k=\max (0,-l)}^{\infty} \frac{(k+l) !}{k !} w^{k} \\
& = \begin{cases}\frac{l !}{(1-w)^{l+1}}, & l \geq 0, \\
\frac{(-w)^{-l}+w(1-w)^{-l-1}-(w-1)^{-l-1} \log (1-w)}{(-l-1) !}, & l<0 .\end{cases}
\end{aligned}
$$

(ii) the coefficients $c_{l}\left(z_{1}\right)$ in (44) are given by the formula

$$
c_{l}\left(z_{1}\right)=\pi^{-N-d} \operatorname{det} g\left(z_{1}\right) e^{d \Phi\left(z_{1}\right)} \sum_{j=0}^{l} a_{N-j, l-j} \mathcal{B}_{j}\left(z_{1}\right),
$$

where $a_{m, n}(m \in \mathbb{Z}, n \geqq 0)$ are universal constants with $a_{m 0}=1$, and $\mathcal{B}_{j}$ are the scalar invariants of $g_{i \bar{j}}$ from (24). 
Taking $N=d=1$ and $\Phi\left(z_{1}\right)$ depending only on $\left|z_{1}\right|$ in Engliš' Theorem 3.8, then $\widetilde{\Omega}$ is a complete Reinhardt domain in $\mathbb{C}^{2}$, which implies Nakazawa's Theorem 2.5 ,

$$
\widetilde{K}=\frac{p}{8 \pi^{2}\left|z_{1} z_{2}\right|^{2}}\left(\frac{L_{0}}{\lambda^{3}}+\frac{L_{1}}{\lambda^{2}}+\frac{L_{2}}{\lambda}+\sum_{k=3}^{\infty} L_{k} \lambda^{k-3} \log \lambda\right) .
$$

We need the following lemma of Nakazawa.

Lemma 3.9 ([48, Prop. 0]). Each coefficient $L_{k}$ is a linear combination of

$$
p^{\left(\eta_{1}\right)} \cdots p^{\left(\eta_{2 k}\right)} / p^{k} \quad \text { with } \eta_{1}+\cdots+\eta_{2 k}=2 k .
$$

Namely $L_{k}$ is homogeneous of degree $k$ and order $2 k$.

We can now prove a closed formula of $L_{k}$ by using (41).

Theorem 3.10. Let $k \geq 0$. Define a function $W_{k}(p)$ by

$$
W_{k}(p)=\frac{1}{p^{k}} \sum_{G \in \mathcal{G}^{s s}(k)}^{\text {quasi-strong }} \frac{(-1)^{|V(G)|+n(G)}}{|\operatorname{Aut}(G)|} \prod_{v \in V(G)} h(\operatorname{deg}(v)-2),
$$

where $G$ runs over all quasi-strong (i.e. all connected components are strongly connected) semistable graphs of weight $k$ and $n(G)$ is the number of components of $G$; the function $h$ is defined recursively by

$$
h(1)=p^{\prime}, \quad h(k)=[p \cdot h(k-1)]^{\prime}, k \geq 2 .
$$

Then the coefficients of (47) are given by

$$
L_{k}= \begin{cases}\frac{(2-k) !}{2} W_{k}(p), & 0 \leq k \leq 2, \\ \frac{(-1)^{k}}{2(k-3) !} W_{k}(p), & k \geq 3 .\end{cases}
$$

Proof. In the notations of Theorem 2.5, for $\left(z_{1}, z_{2}\right) \in \mathbb{C}^{2}$, we have

$$
\begin{gathered}
x=-\log \left|z_{1}\right|=-\frac{1}{2}\left(\log z_{1}+\log \bar{z}_{1}\right), \quad y=-\log \left|z_{2}\right|, \quad f(x)=\frac{1}{2} \Phi, \\
e^{-2 \lambda}=\left|z_{2}\right|^{2} e^{\Phi\left(z_{1}\right)}, \quad \frac{\partial^{2} \Phi}{\partial z_{1} \bar{\partial} z_{1}}=\frac{1}{2\left|z_{1}\right|^{2}} \frac{\partial^{2} f}{\partial x^{2}}=\frac{p}{2\left|z_{1}\right|^{2}} .
\end{gathered}
$$

By using these equations, (44) becomes

$$
\widetilde{K}=\frac{1}{\pi^{2}} \frac{p}{2\left|z_{1}\right|^{2}} \frac{e^{-2 \lambda}}{\left|z_{2}\right|^{2}} \sum_{k=0}^{\infty} \sum_{j=0}^{k} a_{1-j, k-j} \mathcal{B}_{j}\left(z_{1}\right) u_{2-k}\left(e^{-2 \lambda}\right) .
$$


By (45), the singular part of $u_{2-k}\left(e^{-2 \lambda}\right)$ is given by

$$
u_{2-k}\left(e^{-2 \lambda}\right)= \begin{cases}\frac{(2-k) !+O(\lambda)}{2^{3-k} \lambda^{3-k}}, & 0 \leq k \leq 2, \\ \frac{\left[(-1)^{k} 2^{k-3} \lambda^{k-3}+O\left(\lambda^{k-2}\right)\right] \log (\lambda)}{(k-3) !}, & k \geq 3 .\end{cases}
$$

Note that the derivatives of $p$ satisfy

$$
\frac{\partial p}{\partial z_{1}}=-\frac{p p^{\prime}}{2 z_{1}}, \quad \frac{\partial p}{\partial \bar{z}_{1}}=-\frac{p p^{\prime}}{2 \bar{z}_{1}}
$$

By (41), we express $\mathcal{B}_{j}\left(z_{1}\right)$ as a summation of rational differential functions of $p$,

$$
\begin{aligned}
\mathcal{B}_{j}\left(z_{1}\right)=\sum_{G \in \mathcal{G}^{s s}(j)}^{\text {quasi-strong }} \frac{(-1)^{|V(G)|+n(G)}}{|\operatorname{Aut}(G)|} & \\
& \times\left.\prod_{v \in V(G)} \frac{\partial^{\operatorname{deg}(v)-2}}{\partial z_{1}^{\operatorname{deg}^{+}(v)-1} \partial \bar{z}_{1}^{\operatorname{deg}^{-}(v)-1}}\left[\frac{p}{2\left|z_{1}\right|^{2}}\right]\right|_{\left|z_{1}\right|^{2}=p / 2}
\end{aligned}
$$

Note that $\mathcal{B}_{j}$ is of degree no more than $j$. The top degree is achieved only when all derivatives are taken on the numerator $p$. It is not difficult to see from (53) that

$$
\begin{aligned}
\mathcal{B}_{j}\left(z_{1}\right) & =\sum_{G \in \mathcal{G}^{s s}(j)}^{\text {quasi-strong }} \frac{(-1)^{|V(G)|+n(G)}}{|\operatorname{Aut}(G)|} \frac{1}{2^{j} p^{j}} \prod_{v \in V(G)} \frac{1}{p} \cdot \frac{\partial^{\operatorname{deg}(v)-2} p}{\partial z_{1}^{\operatorname{deg}^{+}(v)-1} \partial \bar{z}_{1}^{\operatorname{deg}^{-}(v)-1}}+\text { Low } \\
& =\sum_{G \in \mathcal{G}^{s s}(j)}^{\text {quasi-strong }} \frac{(-1)^{|V(G)|+n(G)}}{|\operatorname{Aut}(G)|} \frac{1}{2^{j} p^{j}} \prod_{v \in V(G)} h(\operatorname{deg}(v)-2)+\text { Low } \\
& =\frac{W_{j}(p)}{2^{j}}+\text { Low, }
\end{aligned}
$$

where Low denotes the terms of rational differential functions of $p$ with degree strictly less than $j$, which may be discarded according to Lemma 3.9. It also implies that in the summation (50), we can discard all terms except when $j=k$, i.e. the term $a_{1-k, 0} B_{k}\left(z_{1}\right)=B_{k}\left(z_{1}\right)$. In view of (51), Equation (49) follows immediately.

Remark 3.11. The formula (48) may be reformulated as

$$
W_{k}(p)=\frac{1}{p^{k}} \sum_{H}^{\text {quasi-strong }} \frac{(-1)^{|V(H)|+n(H)}}{|V(H)| !(|V(H)|+k) !} \prod_{v \in V(H)} h(\operatorname{deg}(v)-2),
$$


where $H$ runs over isomorphism classes of labeled semistable quasi-strong graphs of weight $k$, i.e. the vertices and edges of $H$ are labeled by $\{1, \ldots,|V(H)|\}$ and $\{1, \ldots,|E(H)|\}$ respectively. Denote by $N\left(d_{1}, \ldots, d_{j}\right)$ the number of labeled strong graphs with given degree sequence $\left(d_{1}, \ldots, d_{j}\right)$. The computation with (54) will be greatly eased if one can find a recursive formula for $N\left(d_{1}, \ldots, d_{j}\right)$, e.g. using the method of [38].

Example 3.12. Obviously $L_{0}=W_{0}(p)=1$. Note that

$$
\begin{aligned}
& h(1)=p^{\prime}, \quad h(2)=\left(p^{\prime}\right)^{2}+p p^{\prime \prime}, \\
& h(3)=\left(p^{\prime}\right)^{3}+4 p p^{\prime} p^{\prime \prime}+p^{2} p^{(3)} \\
& h(4)=\left(p^{\prime}\right)^{4}+11 p\left(p^{\prime}\right)^{2} p^{\prime \prime}+7 p^{2} p^{\prime} p^{(3)}+4 p^{2}\left(p^{\prime \prime}\right)^{2}+p^{3} p^{(4)} .
\end{aligned}
$$

We now compute $L_{1}, L_{2}, L_{3}$ by using Theorem 3.10. There are two quasi-strong graphs in $\mathcal{G}^{s s}(1)$

$$
\text { [2] }[\circ \stackrel{2}{\underset{1}{2}} 0]
$$

So $W_{1}(p)=\frac{1}{p}\left(\frac{h(2)}{2}-\frac{h(1)^{2}}{2}\right)=\frac{1}{2} p^{\prime \prime}$, which implies $L_{1}=\frac{1}{4} p^{\prime \prime}$.

There are 19 quasi-strong graphs in $\mathcal{G}^{s s}(2)$ as depicted in Table 0, among which 4 are stable. They are grouped according to their stabilization graphs. It is a routine calculation that $W_{2}(p)=\frac{1}{6}\left(p p^{(3)}\right)^{\prime}$, which implies $L_{2}=\frac{1}{12}\left(p p^{(3)}\right)^{\prime}$.

There are 300 quasi-strong graphs in $\mathcal{G}^{s s}(3)$, among which 14 are stable. With the help of Maple, we get $W_{3}(p)=\frac{1}{24}\left(p^{2} p^{(4)}\right)^{\prime \prime}$, which implies $L_{3}=-\frac{1}{48}\left(p^{2} p^{(4)}\right)^{\prime \prime}$.

Now we consider the higher dimensional Reinhardt domains. Let $n \geq 2$. Under the notations of Theorem 2.7, denote

$$
\widetilde{K}=\frac{n ! p_{1} \cdots p_{n-1}}{4^{n} \pi^{n}\left|z_{1} \cdots z_{n}\right|^{2}}\left(\sum_{k=0}^{n} \frac{L_{k}}{\lambda^{n+1-k}}+\sum_{k=n+1}^{\infty} L_{k} \lambda^{k-(n+1)} \log \lambda\right) .
$$

Theorem 3.13. Let $k \geq 0$. Then the coefficients of (55) are given by

$$
L_{k}= \begin{cases}\frac{(n-k) !}{n !} \sum_{\substack{k=m_{1}+\cdots+m_{n-1} \\ m_{i} \geq 0}} \prod_{i=1}^{n-1} W_{m_{i}}\left(p_{i}\right), & 0 \leq k \leq n, \\ \frac{(-1)^{n-k}}{n !(k-1-n) !} \sum_{\substack{k=m_{1}+\cdots+m_{n-1} \\ m_{i} \geq 0}} \prod_{i=1}^{n-1} W_{m_{i}}\left(p_{i}\right), \quad k \geq n+1,\end{cases}
$$


TABLE 0. The 19 quasi-strong semistable graphs of weight 2

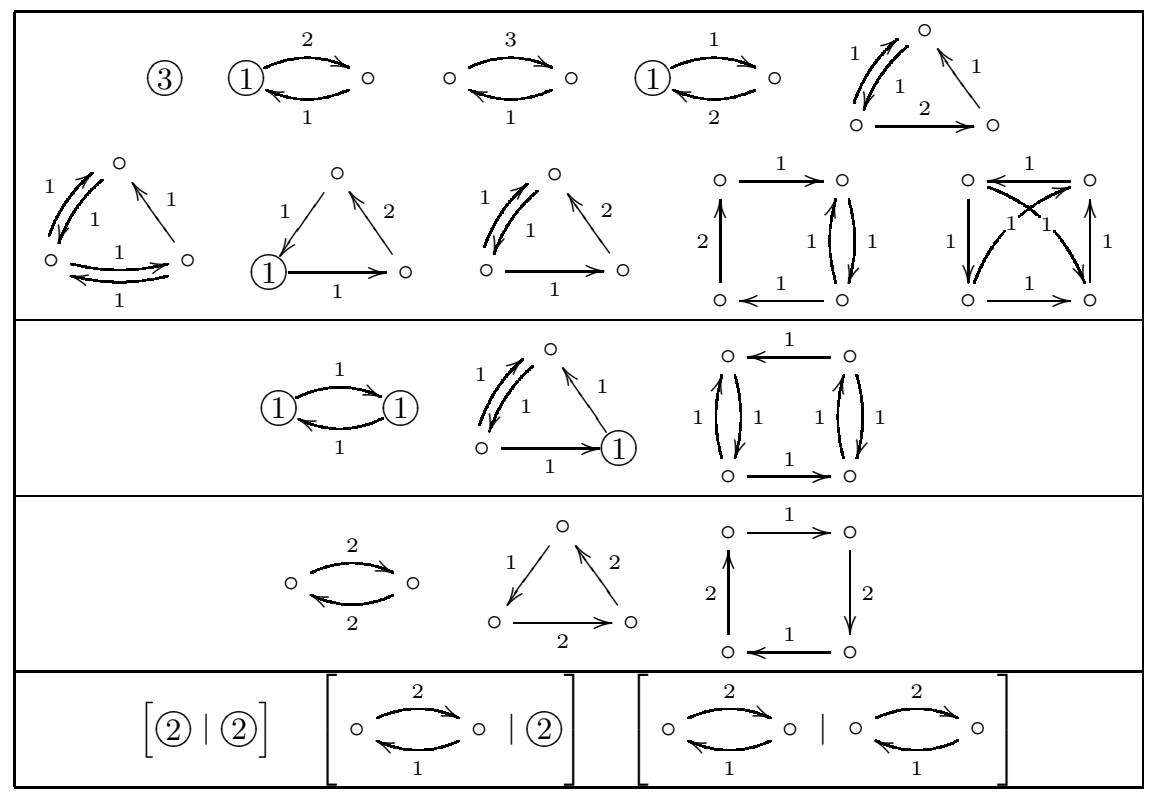

where $W_{m_{i}}\left(p_{i}\right)$ is the function defined in (48) with $p$ replaced by $p_{i}$.

Proof. Under the notations of Theorem 2.7, (44) becomes

$$
\widetilde{K}=\frac{1}{\pi^{n}} \frac{p_{1} \cdot p_{n-1}}{2^{n-1}\left|z_{1} \cdots z_{n-1}\right|^{2}} \frac{e^{-2 \lambda}}{\left|z_{n}\right|^{2}} \sum_{k=0}^{\infty} \sum_{j=0}^{k} a_{n-1-j, k-j} \mathcal{B}_{j}\left(z_{1}\right) u_{n-k}\left(e^{-2 \lambda}\right) .
$$

Note that we have the analogue of Lemma 3.9 for any $n \geq 2$. So we can use the same argument as Theorem 3.10. The singular part of $u_{n-k}\left(e^{-2 \lambda}\right)$ is given by

$$
u_{n-k}\left(e^{-2 \lambda}\right)= \begin{cases}\frac{(n-k) !+O(\lambda)}{2^{n+1-k} \lambda^{n+1-k}}, & 0 \leq k \leq n, \\ \frac{\left[(-1)^{n-k} 2^{k-n-1} \lambda^{k-n-1}+O\left(\lambda^{k-n}\right)\right] \log (\lambda)}{(k-n-1) !}, & k \geq n+1 .\end{cases}
$$

Finally, (56) follows from the multiplicativity of $\mathcal{B}_{k}$.

We can easily compute $L_{1}, L_{2}$ by using (56) and Example 3.12.

$$
L_{1}=\frac{1}{n} \sum_{i=1}^{n-1} W_{1}\left(p_{i}\right)=\frac{1}{2 n} \sum_{i=1}^{n-1} p_{i}^{\prime \prime}
$$




$$
\begin{aligned}
L_{2} & =\frac{1}{n(n-1)}\left(\sum_{i=1}^{n-1} W_{2}\left(p_{i}\right)+\frac{1}{2} \sum_{i \neq j} W_{1}\left(p_{i}\right) W_{1}\left(p_{j}\right)\right) \\
& =\frac{1}{n(n-1)}\left(\frac{1}{6} \sum_{i=1}^{n-1}\left(p_{i} p_{i}^{(3)}\right)^{\prime}+\frac{1}{8} \sum_{i \neq j} p_{i}^{\prime \prime} p_{j}^{\prime \prime}\right),
\end{aligned}
$$

which agree with $(22)$.

\section{From partial to COVARIANT Derivatives}

Definition 4.1. A rooted tree $T$ is an oriented tree with a special vertex $r$, called the root, such that there is a unique directed path from $r$ to any vertex $v$, i.e. all edges point away from $r$. We use $V(T)$ and $E(T)$ to denote the set of vertices and edges of $T$ respectively. A subtree of $T$ is a tree consisting of a vertex in $T$ and all of its descendants. The subtree corresponding to $r$ is $T$; a proper subtree is a subtree corresponding to any other vertex.

The number $T_{n}$ of rooted trees on $n$ nodes can be calculated from the following recursive equation

$$
T_{n+1}=\frac{1}{n} \sum_{i=1}^{n}\left(\sum_{d \mid i} d T_{d}\right) T_{n-i+1},
$$

where $d$ runs over the factors of $i$. In terms of generating functions

$$
T(x)=\sum_{n=1}^{\infty} T_{n} x^{n}=x+x^{2}+2 x^{3}+4 x^{4}+9 x^{5}+20 x^{6}+48 x^{7}+115 x^{8}+\cdots .
$$

Asymptotically $T_{n}$ satisfies

$$
T_{n} \sim \frac{c}{n^{3 / 2}} \eta^{-n}, \quad n \rightarrow \infty,
$$

where $\eta=0.3383 \ldots$ is the radius of convergence of $T(x)$ and $c=0.4399 \ldots$

For trees in Figure 1, the root is either the leftmost or bottommost vertex.

Definition 4.2. A decorated tree $T$ is a directed tree such that each vertex is decorated by a finite number of outward and inward external legs, corresponding to unbarred and barred indices respectively. $T$ is called stable if each vertex is stable, i.e. has at least two outward half-edges and two inward half-edges. Note that a half-edge may refer to the head or tail of an edge of $T$ or an external leg. 


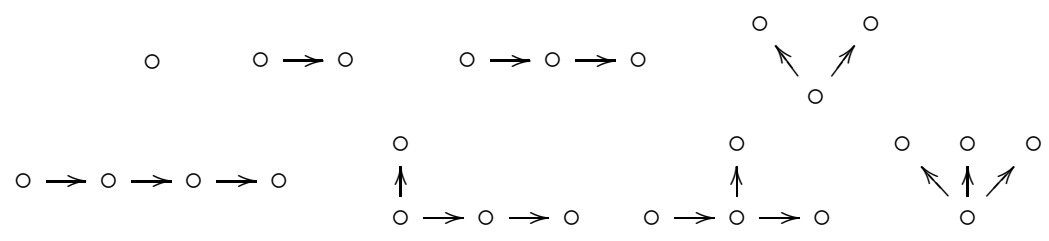

FiguRE 1. Rooted trees with no more than 4 vertices

Let $(T, r)$ be a stable rooted tree decorated by $\left\{a_{1} \cdots a_{k}, \bar{b}_{1} \cdots \bar{b}_{m}\right\}$. For each vertex $v$ of $T$, we define $i(v)=\min \left\{k \mid \bar{b}_{k} \in v\right\}$, which is well-defined since any vertex of $T$ has at most one inward edge.

Definition 4.3. Denote by $\mathscr{T}_{R}\left(a_{1} \cdots a_{k} \mid \bar{b}_{1} \cdots \bar{b}_{m}\right)$ the set of all decorated stable rooted trees $T$ with root $r$ such that $\bar{b}_{1}, \bar{b}_{2} \in r$ and for each directed edge $u v$, we have $i(u)<i(v)$.

Define $\mathscr{T}_{R}\left(a_{1} \cdots a_{p}\left|a_{p+1} \cdots a_{k}\right| \bar{b}_{1} \cdots \bar{b}_{m}\right)$ to be the subset of $\mathscr{T}_{R}\left(a_{1} \cdots a_{k} \mid \bar{b}_{1} \cdots \bar{b}_{m}\right)$ containing trees with the property that there does not exist a proper subtree which contains at least two indices from $\left\{a_{1}, \ldots, a_{p}\right\}$. Its complement is denoted by $\mathscr{T}_{R}^{c}\left(a_{1} \cdots a_{p}\left|a_{p+1} \cdots a_{k}\right| \bar{b}_{1} \cdots \bar{b}_{m}\right)$. The enumeration problem of these trees will be discussed in Appendix B.

Similar to the definition of Weyl invariants, for each $T \in \mathscr{T}_{R}\left(a_{1} \cdots a_{k} \mid \bar{b}_{1} \cdots \bar{b}_{m}\right)$, we associate a unique monomial of curvature tensors $R_{T}$ given by

$$
R_{T}=(-1)^{|V(T)|} \prod_{e \in E(T)} g^{a_{e} \bar{b}_{e}} \prod_{v \in V(T)} R_{v},
$$

where the indices $a_{e}, \bar{b}_{e}$ correspond to the head and tail of $e$ respectively and $R_{v}$ is the curvature tensor obtained by arranging the indices of half-edges of $v$ as follows

$$
R_{v}= \begin{cases}R_{a_{*} \bar{b}_{1} a_{*} \bar{b}_{2} / a_{*} \cdots a_{*} \bar{b}_{*} \cdots \bar{b}_{*},}, & v=r, \\ R_{a_{*} \bar{b}_{i(v)} a_{*} \bar{b}_{e} / a_{*} \cdots a_{*} \bar{b}_{*} \cdots \bar{b}_{*},}, v \neq r .\end{cases}
$$

Note that when $v \neq r, \bar{b}_{e}$ corresponds to the tail of the unique inward edge $e \in E(T)$ of $v$. By the second Bianchi identity (5) and the Ricci formula (6), $R_{a_{*} \bar{b}_{1} a_{*} \bar{b}_{2} / a_{*} \cdots a_{*} \bar{b}_{*} \cdots \bar{b}_{*}}$ is separately symmetric in the indices $a_{*}$ and $\bar{b}_{*}$, so their orders are irrelevant and thus $R_{T}$ is well-defined.

The following theorem is one of the main results of this paper. 
Theorem 4.4. Let $k \geq p \geq 2$ and $m \geq 2$. Then

$$
\begin{gathered}
D\left(\left(g^{r \bar{s}} g_{r \bar{b}_{1} \bar{b}_{2}} g_{a_{1} \bar{s} a_{2}}\right)_{a_{3} \cdots a_{k} \bar{b}_{3} \cdots \bar{b}_{m}}\right)= \\
-D\left(\left(R_{a_{1} \bar{b}_{1} a_{2} \bar{b}_{2} / a_{3} \cdots a_{p}}\right)_{a_{p+1} \cdots a_{k} \bar{b}_{3} \cdots \bar{b}_{m}}\right)=\sum_{T \in \mathscr{T}_{R}^{c}\left(a_{1} a_{2}\left|a_{3} \cdots a_{k}\right| \bar{b}_{1} \cdots \bar{b}_{m}\right)} R_{T}, \sum_{T \in \mathscr{T}_{R}\left(a_{1} \cdots a_{p}\left|a_{p+1} \cdots a_{k}\right| \bar{b}_{1} \cdots \bar{b}_{m}\right)} R_{T}, \\
D\left(g_{\left.a_{1} \bar{b}_{1} a_{2} \bar{b}_{2} a_{3} \cdots a_{k} \bar{b}_{3} \cdots \bar{b}_{m}\right)}=\sum_{T \in \mathscr{T}_{R}\left(a_{1} \cdots a_{k} \mid \bar{b}_{1} \cdots \bar{b}_{m}\right)} R_{T} .\right.
\end{gathered}
$$

Proof. First the theorem is obviously true when $k=m=2$ or $p=k$. We will proceed by induction.

First we prove (61). Note that when applying $\partial_{a_{3} \cdots a_{k} \bar{b}_{3} \cdots \bar{b}_{m}}$ to $g^{r \bar{s}} g_{r \bar{b}_{1} \bar{b}_{2}} g_{a_{1} \bar{s} a_{2}}$, i.e. the decorated tree

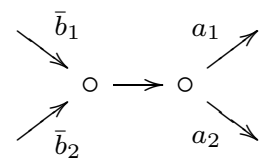

we get a sum of paths decorated by $\left\{a_{3} \cdots a_{k} \bar{b}_{3} \cdots \bar{b}_{m}\right\}$. By induction, we may use (63) to convert the above partial derivatives to covariant derivatives and get a sum of decorated trees $(T, r)$ with the property that $a_{1}, a_{2}$ are contained in a proper subtree $T^{\prime}$ of $T$. We need to compute the coefficient of $T$ in the tensorial polynomial $D\left(\left(g^{r \bar{s}} g_{r \bar{b}_{1} \bar{b}_{2}} g_{a_{1} \bar{s} a_{2}}\right)_{a_{3} \cdots a_{k} \bar{b}_{3} \cdots \bar{b}_{m}}\right)$. It is without loss of generality to contract $T^{\prime}$ to a single vertex, so we may assume that $a_{1}, a_{2}$ are attached to the same vertex $v$. Let $P_{n}, n \geq 2$ be the unique path $\left(r=v_{1}, v_{2}, \ldots, v_{n-1}, v_{n}=v\right)$ from $r$ to $v$. If $T \in \mathscr{T}_{R}^{c}\left(a_{1} a_{2}\left|a_{3} \cdots a_{k}\right| \bar{b}_{1} \cdots \bar{b}_{m}\right)$, i.e. $i(u)<i(v)$ whenever $u v \in$ $E(T)$, the coefficient of $T$ in $D\left(\left(g^{r \bar{s}} g_{r \bar{b}_{1} \bar{b}_{2}} g_{a_{1} \bar{s} a_{2}}\right)_{a_{3} \cdots a_{k} \bar{b}_{3} \cdots \bar{b}_{m}}\right)$ is equal to

$$
\sum_{j=1}^{n-1}\left(\begin{array}{c}
n-1 \\
j
\end{array}\right)(-1)^{j+1}=1 .
$$

On the other hand, if there are $k \geq 1$ edges $u v$ in $P_{n}$ that violate $i(u)<i(v)$, the coefficient of $T$ in $D\left(\left(g^{r \bar{s}} g_{r \bar{b}} \bar{b}_{1} g_{a_{1}} \bar{s} a_{2}\right)_{a_{3} \cdots a_{k} \bar{b}_{3} \cdots \bar{b}_{m}}\right)$ is equal to

$$
\sum_{j=0}^{n-1-k}\left(\begin{array}{c}
n-1-k \\
j
\end{array}\right)(-1)^{j+k+1}=0,
$$

which follows from $k<n-1$. So we conclude the inductive proof of (61). 
Next we prove (62). Note that (62) holds when $p=k$ by (8). From (2), we have

$$
\begin{aligned}
& \left(R_{a_{1} \bar{b}_{1} a_{2} \bar{b}_{2} / a_{3} \cdots a_{p}}\right)_{a_{p+1} \cdots a_{k} \bar{b}_{3} \cdots \bar{b}_{m}}=\left(R_{a_{1} \bar{b}_{1} a_{2} \bar{b}_{2} / a_{3} \cdots a_{p} a_{p+1}}\right)_{a_{p+2} \cdots a_{k} \bar{b}_{3} \cdots \bar{b}_{m}} \\
& +\left(g^{r \bar{s}} g_{a_{p+1} \bar{s} a_{1}} R_{r \bar{b}_{1} a_{2} \overline{b_{2}} / a_{3} \cdots a_{p}}+g^{r \bar{s}} g_{a_{p+1} \bar{s} a_{2}} R_{a_{1} \bar{b}_{1} r \bar{b}_{2} / a_{3} \cdots a_{p}}\right)_{a_{p+2} \cdots a_{k} \bar{b}_{3} \cdots \bar{b}_{m}} \\
& +\sum_{j=3}^{p}\left(g^{r \bar{s}} g_{a_{p+1} \bar{s} a_{j}} R_{a_{1} \bar{b}_{1} a_{2} \bar{b}_{2} / a_{3} \cdots a_{j-1} r a_{j+1} \cdots a_{p}}\right)_{a_{p+2} \cdots a_{k} \bar{b}_{3} \cdots \bar{b}_{m}}
\end{aligned}
$$

Then by induction, in the right-hand side of (64), the first term produces trees with the property that no two of $\left\{a_{1}, \ldots, a_{p}, a_{p+1}\right\}$ are contained in a proper subtree, the second term produces trees with the property that either $a_{1}, a_{p+1}$ or $a_{2}, a_{p+1}$ are contained in a proper subtree, the last term produces trees with the property that $a_{j}, a_{p+1}(3 \leq j \leq p)$ are contained in a proper subtree. The coefficient of each tree can be determined by exactly the same argument as in the above proof of (61). So we conclude the inductive proof of (62).

Finally we prove (63). By (1), we have

$$
g_{a_{1} \bar{b}_{1} a_{2} \bar{b}_{2} a_{3} \cdots a_{k} \bar{b}_{3} \cdots \bar{b}_{m}}=-\left(R_{a_{1} \bar{b}_{1} a_{2} \bar{b}_{2}}\right)_{a_{3} \cdots a_{k} \bar{b}_{3} \cdots \bar{b}_{m}}+\left(g^{r \bar{s}} g_{r \bar{b}_{1} \bar{b}_{2}} g_{a_{1} \bar{s} a_{2}}\right)_{a_{3} \cdots a_{k} \bar{b}_{3} \cdots \bar{b}_{m}} .
$$

So the expression of $D\left(g_{a_{1}} \bar{b}_{1} a_{2} \bar{b}_{2} a_{3} \cdots a_{k} \bar{b}_{3} \cdots \bar{b}_{m}\right)$ in (63) follows directly from (61) and the $p=2$ case of (62). Therefore we conclude the inductive proof of the theorem.

Remark 4.5. See [54] for related results more general than Theorem 4.4.

We sort indices in alphabetical order, $i, j, k, l, p, q, \ldots$ Note that only the order of barred indices is essential.

Example 4.6. Let us compute $D\left(g_{i \bar{j} k \bar{l} p \bar{q}}\right)$. Besides the one-vertex tree, there are three two-vertex trees in $\mathscr{T}_{R}(i k p \mid \bar{j} \bar{l} \bar{q})$,
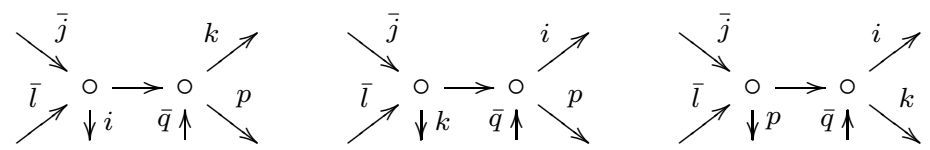

By (63), we get

$$
D\left(g_{i \bar{j} k \bar{l} p \bar{q}}\right)=-R_{i \bar{j} k \bar{l} / p \bar{q}}+g^{s \bar{t}}\left(R_{i \bar{j} s \bar{l}} R_{k \bar{q} p \bar{t}}+R_{k \bar{j} s \bar{l}} R_{i \bar{q} p \bar{t}}+R_{p \bar{j} s \bar{l}} R_{i \bar{q} k \bar{t}}\right) .
$$

Example 4.7. Since both $\mathscr{T}_{R}\left(i k p_{1} \ldots p_{r} \mid \bar{j} \bar{l}\right)$ and $\mathscr{T}_{R}\left(i k \mid \bar{j} \bar{l} \bar{p}_{1} \ldots \bar{p}_{r}\right)$ contain only one tree, we get $D\left(g_{i \bar{j} k \bar{l} p_{1} \ldots p_{r}}\right)=-R_{i \bar{j} k \bar{l} / p_{1} \ldots p_{r}}$ and $D\left(g_{i \bar{j} k \bar{p} \bar{p}_{1} \ldots \bar{p}_{r}}\right)=-R_{i \bar{j} k \bar{l} / \bar{p}_{1} \ldots \bar{p}_{r}}$. 
The next theorem shows the difference when we interchange two non-adjacent barred indices in a curvature tensor.

Theorem 4.8. Let $m \geq 3$. Then

$$
R_{a_{1} \bar{b}_{1} a_{2} \bar{b}_{2} / a_{3} \cdots a_{k} \bar{b}_{3} \cdots \bar{b}_{m}}-R_{a_{1} \bar{b}_{1} a_{2} \bar{b}_{3} / a_{3} \cdots a_{k} \bar{b}_{2} \bar{b}_{4} \cdots \bar{b}_{m}}=\sum_{T} \eta(T) R_{T}
$$

where $T=\{u \stackrel{e}{\rightarrow} v\}$ runs over stable two-vertex paths decorated by indices $\left\{a_{1} \cdots a_{k}, \bar{b}_{1} \cdots \bar{b}_{m}\right\}$ and

$$
\eta(T)= \begin{cases}1, & \text { if } \bar{b}_{1}, \bar{b}_{2} \in u \text { and } \bar{b}_{3} \in v, \\ -1, & \text { if } \bar{b}_{1}, \bar{b}_{3} \in u \text { and } \bar{b}_{2} \in v \\ 0, & \text { otherwise. }\end{cases}
$$

The curvature tensor $R_{T}$ corresponding to $T$ is given by (cf. (80))

$$
R_{T}= \begin{cases}R_{a_{*} \bar{b}_{1} a_{*} \bar{b}_{2} / a_{*} \cdots a_{*} \bar{b}_{*} \cdots \bar{b}_{*}} R_{a_{*} \bar{b}_{3} a_{*} \bar{b}_{e} / a_{*} \cdots a_{*} \bar{b}_{*} \cdots \bar{b}_{*},} & \text { if } \bar{b}_{1}, \bar{b}_{2} \in u \text { and } \bar{b}_{3} \in v, \\ R_{a_{*} \bar{b}_{1} a_{*} \bar{b}_{3} / a_{*} \cdots a_{*} \bar{b}_{*} \cdots \bar{b}_{*}} R_{a_{*} \bar{b}_{2} a_{*} \bar{b}_{e} / a_{*} \cdots a_{*} \bar{b}_{*} \cdots \bar{b}_{*},}, \quad \text { if } \bar{b}_{1}, \bar{b}_{3} \in u \text { and } \bar{b}_{2} \in v .\end{cases}
$$

Proof. The left-hand side of (66) is equal to

$$
\begin{aligned}
& \sum_{j=3}^{k}\left(R_{a_{1} \bar{b}_{1} a_{2} \bar{b}_{2} / a_{3} \cdots a_{j} \bar{b}_{3} a_{j+1} \cdots a_{k} \bar{b}_{4} \cdots \bar{b}_{m}}-R_{\left.a_{1} \bar{b}_{1} a_{2} \bar{b}_{2} / a_{3} \cdots a_{j-1} \bar{b}_{3} a_{j} \cdots a_{k} \bar{b}_{4} \cdots \bar{b}_{m}\right)}\right. \\
& \quad-\sum_{j=3}^{k}\left(R_{a_{1} \bar{b}_{1} a_{2} \bar{b}_{3} / a_{3} \cdots a_{j} \bar{b}_{2} a_{j+1} \cdots a_{k} \bar{b}_{4} \cdots \bar{b}_{m}}-R_{a_{1} \bar{b}_{1} a_{2} \bar{b}_{3} / a_{3} \cdots a_{j-1} \bar{b}_{2} a_{j} \cdots a_{k} \bar{b}_{4} \cdots \bar{b}_{m}}\right)
\end{aligned}
$$

By the Ricci formula (7), it is not difficult to see that any two-vertex path $T=$ $\{u \stackrel{e}{\rightarrow} v\}$ appearing in (68) must satisfy one of the following conditions:

(i) $\bar{b}_{1}, \bar{b}_{2} \in u, \bar{b}_{3} \in v$; (ii) $\bar{b}_{1}, \bar{b}_{3} \in u, \bar{b}_{2} \in v$; (iii) $\bar{b}_{2}, \bar{b}_{3} \in u, \bar{b}_{1} \in v$.

Again by the Ricci formula (7), the contributions of the two summations in (68) to (iii) cancel out.

A two-vertex path $T$ in (i) is contained in the first (resp. second) summation in (68) if and only if at least one of $a_{1}, a_{2}$ belongs to $u$ (resp. both $a_{1}, a_{2}$ belong to $v)$.

A two-vertex path $T$ in (ii) is contained in the first (resp. second) summation in (68) if and only if both $a_{1}, a_{2}$ belong to $v$ (resp. at least one of $a_{1}, a_{2}$ belongs to $u)$. 
Finally, (68) follows by noting that $T$ in (i) and (ii) has multiplicity 1 and -1 respectively.

Example 4.9. By (66), we have

$$
\begin{aligned}
& R_{i \bar{j} k \bar{l} / p \bar{q}}=R_{i \bar{j} k \bar{q} / p \bar{l}}
\end{aligned}
$$

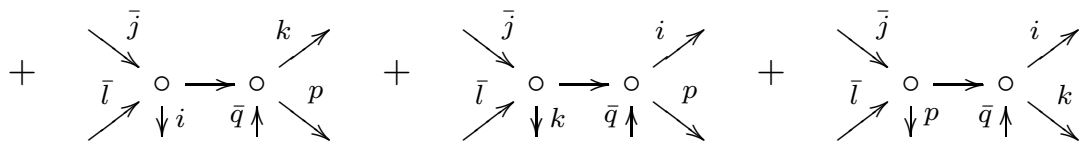

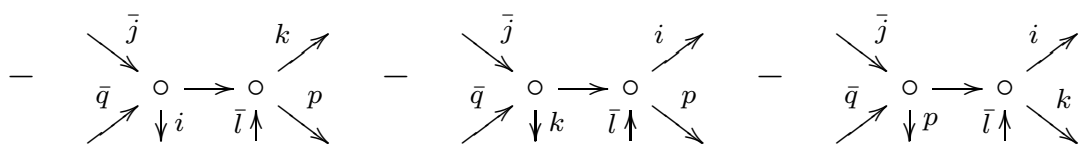

$$
\begin{aligned}
& =R_{i \bar{j} k \bar{q} / p \bar{l}}+R_{i \bar{j} s \bar{l}} R_{k \bar{q} p \bar{s}}+R_{k \bar{j} s \bar{l}} R_{i \bar{q} p \bar{s}}+R_{p \bar{j} s \bar{l}} R_{i \bar{q} k \bar{s}} \\
& -R_{i \bar{j} s \bar{q}} R_{k \bar{l} \bar{p} \bar{s}}-R_{k \bar{j} s \bar{q}} R_{i \bar{l} p \bar{s}}-R_{p \bar{j} s \bar{q}} R_{i \bar{l} k \bar{s}} .
\end{aligned}
$$

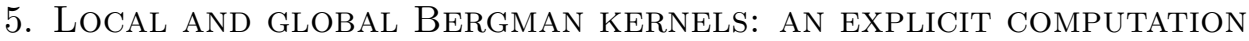

First we briefly review the definitions of Bergman kernel in both local and global settings. Let $\Omega$ be an open subset of $\mathbb{C}^{n}$ with a Kähler potential $\Phi(x)$. Let $\Phi(x, y)$ be an almost analytic extension of $\Phi(x)$ to a neighborhood of the diagonal, i.e. $\bar{\partial}_{x} \Phi$ and $\partial_{y} \Phi$ vanish to infinite order for $x=y$. We can assume $\overline{\Phi(x, y)}=$ $\Phi(y, x)$. For $m>0$, consider the weighted Bergman space of all holomorphic functions on $\Omega$ square-integrable with respect to the measure $e^{-m \Phi} \frac{\omega_{g}^{n}}{n !}$. We denote by $K_{m}(x, y)$ the reproducing kernel. When $\Omega$ is a strongly pseudoconvex domain with real analytic boundary, Engliš [18] proved the existence of the asymptotic expansion

$$
K_{m}(x, y) \sim e^{m \Phi(x, y)} \sum_{k=0}^{\infty} B_{k}(x, y) m^{n-k}, \quad m \rightarrow \infty,
$$

uniformly on compact subsets. The asymptotic expansion (70) plays a crucial role in Berezin quantization [5]. The coefficients $B_{k}$ along the diagonal were computed by Engliš [17] for $k \leq 3$ by a recursive formula of $B_{k}$ derived from the asymptotics of Laplace integrals. Loi $[43,44]$ refined Engliš' recursive formula and gave a new proof of Engliš' asymptotic expansion. See also [10] for related 
works. As shown in [43] (also cf. $[51, \S 3]$ ), $B_{k}$ is equal to $a_{k}$ of $(71)$ in the global case.

For a compact Kähler manifold $M$ of dimension $n$, instead of holomorphic functions, we consider holomorphic sections of holomorphic line bundles. Let $(L, h) \rightarrow M$ be a positive Hermitian holomorphic line bundle and $g$ be the polarized Kähler metric on $M$ corresponding to the Kähler form $\omega_{g}=\operatorname{Ric}(h)$. For each $m \in \mathbb{N}, h$ induces a Hermitian metric $h_{m}$ on $L^{m}$. Let $\left\{S_{1}, \cdots, S_{d}\right\}$ be an orthonormal basis of $H^{0}\left(M, L^{m}\right)$ with respect to the inner product

$$
\left(S_{i}, S_{j}\right)_{h_{m}}=\int_{M} h_{m}\left(S_{i}(x), S_{j}(x)\right) \frac{1}{n !} \omega_{g}^{n} .
$$

Zelditch [57] and Catlin [9] independently proved that there is a complete asymptotic expansion:

$$
\sum_{i=0}^{d}\left\|S_{i}(x)\right\|_{h_{m}}^{2}=a_{0}(x) m^{n}+a_{1}(x) m^{n-1}+a_{2}(x) m^{n-2}+\cdots
$$

Various extensions to off-diagonal asymptotic expansion and generalizations to orbifolds and symplectic manifolds can be found in e.g. [6, 13, 14, 35, 46, 49]. See also recent works $[2,16,24,33,42]$.

The $a_{k}$ for $k \leq 3$ were computed by Lu [45] using peak section method. In particular,

$$
a_{0}=1, \quad a_{1}=\frac{\rho}{2}, \quad a_{2}=\frac{1}{3} \Delta \rho+\frac{1}{24}|R|^{2}-\frac{1}{6}|R i c|^{2}+\frac{1}{8} \rho^{2} .
$$

The computation of $a_{3}$, independently done by Lu [45] and Engliš [17], is a marvelous feat; this requires technical and hard calculations occupying more than ten pages in both papers.

In the rest of the section, we use results proved in $\S 4$ to give a relatively easy derivation of the tensor expression for $a_{3}$. The following explicit closed formula of $a_{k}$ was proved in $[51,52]$,

$$
B_{k}(x)=a_{k}(x)=\sum_{G} z(G) \cdot G=\sum_{G} \frac{(-1)^{n} \operatorname{det}(A-I)}{|\operatorname{Aut}(G)|} G,
$$

where $G=G_{1} \cup \cdots \cup G_{n}$ runs over stable (i.e. both the indegree and outdegree of each vertex are no less than 2) multi-digraphs of weight $k$ (i.e. $|E(G)|-|V(G)|=$ $k$ ) such that each component $G_{i}$ is strongly connected and $A$ is the adjacency matrix of $G$. Although (72) gives a closed formula of $a_{k}$ as a summation of local 
partial derivatives, it is still a quite hard task to convert it into tensor expressions when $k \geq 3$.

We will express $a_{3}$ in terms of the following basis as used by Engliš [17].

$$
\begin{aligned}
& \sigma_{1}=\rho^{3}, \quad \sigma_{2}=\rho R_{i \bar{j}} R_{\bar{j} \bar{i}}, \quad \sigma_{3}=\rho R_{i \bar{j} k \bar{l}} R_{\bar{j} \bar{i} \bar{l} \bar{k}}, \\
& \sigma_{4}=R_{i \bar{j}} R_{k \bar{l}} R_{j \bar{i} l \bar{k}}, \quad \sigma_{5}=R_{i \bar{j}} R_{k \bar{i} l \bar{m}} R_{j \bar{k} m \bar{l}}, \quad \sigma_{6}=R_{i \bar{j}} R_{j \bar{k}} R_{k \bar{i}}, \\
& \sigma_{7}=R_{i \bar{j} k \bar{l}} R_{j \bar{i} \bar{m} \bar{n}} R_{l \bar{k} n \bar{m}}, \quad \sigma_{8}=\rho \Delta \rho, \quad \sigma_{9}=R_{i \bar{j}} R_{j \bar{i} / k \bar{k}}, \\
& \sigma_{10}=R_{i \bar{j} k \bar{l}} R_{j \bar{i} l \bar{k} / m \bar{m}}, \quad \sigma_{11}=\rho_{/ i} \rho_{/ \bar{i}}, \quad \sigma_{12}=R_{i \bar{j} / k} R_{j \bar{i} / \bar{k}}, \\
& \sigma_{13}=R_{i \bar{j} k \bar{l} / m} R_{j \bar{i} l \bar{k} / \bar{m}}, \quad \sigma_{14}=\Delta^{2} \rho, \quad \sigma_{15}=R_{i \bar{j} k \bar{l}} R_{j \bar{m} l \bar{n}} R_{m \bar{i} n \bar{k}} .
\end{aligned}
$$

We need two more tensors

$$
\widetilde{\sigma}_{9}=R_{i \bar{j}} \rho_{\bar{j} \bar{i}}, \quad \widetilde{\sigma}_{10}=R_{i \bar{j} k \bar{l}} R_{j \bar{i} / l \bar{k}} .
$$

By (69), it is not difficult to get

$$
\tilde{\sigma}_{9}=\sigma_{9}+\sigma_{4}-\sigma_{6}, \quad \tilde{\sigma}_{10}=\sigma_{10}+2 \sigma_{7}-\sigma_{5}-\sigma_{15} .
$$

We will compute the coefficients $c_{i}, 1 \leq i \leq 15$, such that

$$
a_{3}=c_{1} \sigma_{1}+c_{2} \sigma_{2}+\cdots+c_{15} \sigma_{15}
$$

There are 15 stable graphs of weight 3 in $\mathcal{G}(3)$.

$$
\begin{aligned}
& \tau_{1}=[(2 \mid 2) \mid 2], \quad \tau_{2}=\left[1 \underset{1}{\frac{1}{2}}(1) \mid 2\right], \quad \tau_{3}=[\stackrel{2}{\stackrel{2}{\rightleftarrows}} \mid 2] \text {, }
\end{aligned}
$$

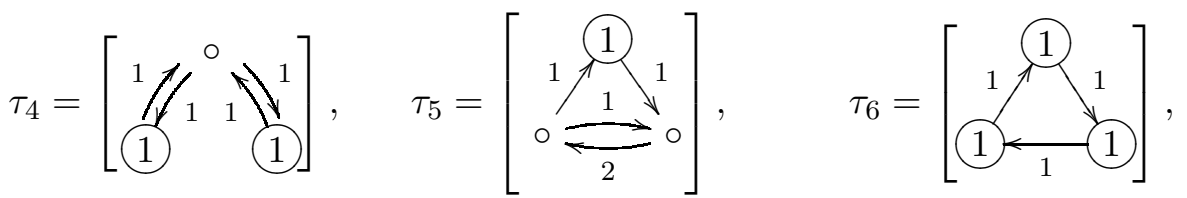

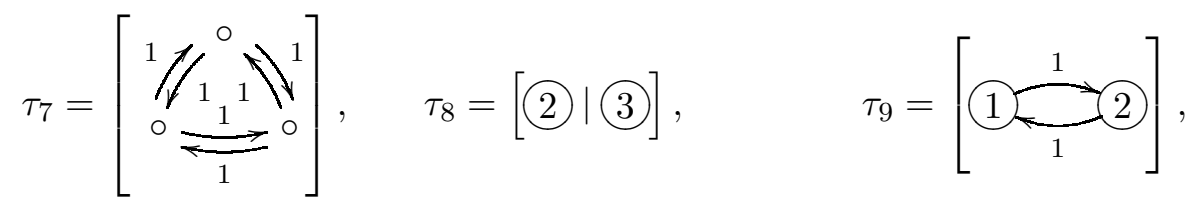

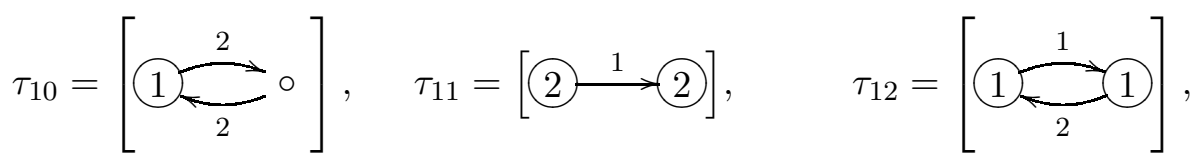




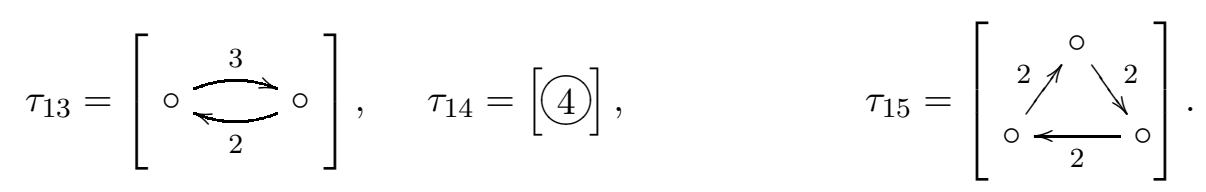

The graphs $\tau_{i}$ are almost in one-to-one correspondence with $\sigma_{i}$. The only exceptions are that $\tau_{9}$ may correspond to either $\sigma_{9}$ or $\widetilde{\sigma}_{9}$, and $\tau_{10}$ may correspond to either $\sigma_{10}$ or $\tilde{\sigma}_{10}$.

By (72), we have

$$
a_{3}=\sum_{i=1}^{15} z\left(\tau_{i}\right) \tau_{i}=z_{1} \tau_{1}+z_{2} \tau_{2}+\cdots+z_{15} \tau_{15},
$$

where the coefficients are given by

$$
\begin{aligned}
& z_{1}=-1 / 48, \quad z_{2}=-1 / 4, \quad z_{3}=-3 / 16, \quad z_{4}=0, \quad z_{5}=-1, \\
& z_{6}=-1 / 3, \quad z_{7}=-2 / 3, \quad z_{8}=1 / 6, \quad z_{9}=1 / 2, \quad z_{10}=1, \\
& z_{11}=0, \quad z_{12}=1, \quad z_{13}=5 / 12, \quad z_{14}=-1 / 8, \quad z_{15}=-7 / 24 \text {. }
\end{aligned}
$$

We need to express each $\tau_{i}$ representing partial derivatives as a linear combination of $\sigma_{i}, 1 \leq i \leq 15$. As stated in [51] without detailed computations,

$$
\begin{gathered}
\tau_{i}=-\sigma_{i}, \quad 1 \leq i \leq 7, \\
\tau_{8}=-2 \sigma_{2}-\sigma_{3}+\sigma_{8}, \quad \tau_{9}=-\sigma_{4}-\sigma_{5}-\sigma_{6}+\sigma_{9}, \quad \tau_{10}=-2 \sigma_{5}+\sigma_{10}-\sigma_{15}, \\
\tau_{11}=\sigma_{11}, \quad \tau_{12}=\sigma_{12}, \quad \tau_{13}=\sigma_{13}, \\
\tau_{14}=-3 \sigma_{4}-12 \sigma_{5}-3 \sigma_{6}+6 \sigma_{7}+7 \sigma_{9}+8 \sigma_{10}+10 \sigma_{12}+3 \sigma_{13}-\sigma_{14}-6 \sigma_{15}, \\
\tau_{15}=-\sigma_{15} .
\end{gathered}
$$

While the terms $\tau_{8}, \tau_{9}, \tau_{10}$ can be computed easily using (65), the computation for $\tau_{14}$ is much more difficult. Here we present a graph-theoretic computation of $\tau_{14}$ by using Theorem 4.4. By (63), the unique one-vertex tree in $D\left(g_{i \bar{i} j \bar{j} k \bar{k} l \bar{l}}\right)$ is equal to

$$
\begin{aligned}
-\rho_{/ k l \bar{k} \bar{l}} & =-\left(\rho_{/ k \bar{k} l \bar{l}}+R_{k \bar{s} l \bar{k} / l} \rho_{/ s}+R_{k \bar{s} l \bar{k}} \rho_{/ s \bar{l}}\right) \\
& =-\left(\sigma_{14}+\sigma_{11}+\widetilde{\sigma}_{9}\right) \\
& =-\left(\sigma_{14}+\sigma_{11}+\sigma_{9}+\sigma_{4}-\sigma_{6}\right)
\end{aligned}
$$

where we used the Ricci formula (7) in the first equation. 
The 48 trees in $D\left(g_{i \bar{i} j \bar{j} k l \bar{k} \bar{l}}\right)$ with at least two vertices are enumerated in Tables 1-6 in Appendix A. Note that each tree $T$ is weighted by $(-1)^{|V(T)|}$. A tree is labeled by the dagger symbol $\uparrow$ if and only if $i, j$ are contained in a proper subtree.

From (1), we have

$$
\tau_{14}=D\left(g_{i \bar{i} j \bar{j} k l \bar{k} \bar{l}}\right)=D\left(-\partial_{k l \bar{k} l} R_{i \bar{i} j \bar{j}}\right)+D\left(\partial_{k l \bar{k} \bar{l}}\left(g^{m \bar{n}} g_{m \bar{i} \bar{j}} g_{i \bar{n} j}\right)\right) .
$$

Denote by $I, I I, \ldots, I V$ the sum of trees in the same numbered table such that $i, j$ are not both contained in a proper subtree. By (62) and (78),

$$
\begin{gathered}
D\left(-\partial_{k l i \bar{k} \bar{l}} R_{i \bar{i} \bar{j}}\right)=-\left(\sigma_{14}+\sigma_{11}+\sigma_{9}+\sigma_{4}-\sigma_{6}\right)+I+I I+I I I+I V+V+V I \\
=-\left(\sigma_{14}+\sigma_{11}+\sigma_{9}+\sigma_{4}-\sigma_{6}\right)+2 \sigma_{9}+\left(\sigma_{11}+4 \sigma_{12}\right)+4 \sigma_{12} \\
+\left(4 \sigma_{9}+2 \widetilde{\sigma}_{9}+4 \widetilde{\sigma}_{10}\right)-\left(2 \sigma_{4}+2 \sigma_{5}+2 \sigma_{6}\right)-\left(2 \sigma_{4}+2 \sigma_{7}\right) \\
=-3 \sigma_{4}-6 \sigma_{5}-3 \sigma_{6}+6 \sigma_{7}+7 \sigma_{9}+4 \sigma_{10}+8 \sigma_{12}-\sigma_{14}-4 \sigma_{15} .
\end{gathered}
$$

Denote by $I^{\prime}, I I^{\prime}, \ldots, I V^{\prime}$ the sum of trees in the same numbered table such that $i, j$ are contained in a proper subtree. By (61),

$$
\begin{aligned}
D\left(\partial_{k l \bar{k} \bar{l}}\left(g^{m \bar{n}} g_{m \bar{i} \bar{j}} g_{i \bar{n} j}\right)\right)=I^{\prime}+I I^{\prime}+I I I^{\prime} & +I V^{\prime}+V^{\prime}+V I^{\prime} \\
=\left(\sigma_{10}+\widetilde{\sigma}_{10}\right)+\sigma_{13}+\left(2 \sigma_{12}+2 \sigma_{13}\right) & +2 \sigma_{10}-\left(3 \sigma_{5}+2 \sigma_{7}+\sigma_{15}\right)-2 \sigma_{5} \\
& =-6 \sigma_{5}+4 \sigma_{10}+2 \sigma_{12}+3 \sigma_{13}-2 \sigma_{15} .
\end{aligned}
$$

Adding up the above two identities, we get the desired tensor expression for $\tau_{14}$. Substituting (77) into (75), we can get the coefficients in (74).

$$
\begin{aligned}
& c_{1}=1 / 48, \quad c_{2}=-1 / 12, \quad c_{3}=1 / 48, \quad c_{4}=-1 / 8, \quad c_{5}=0, \\
& c_{6}=5 / 24, \quad c_{7}=-1 / 12, \quad c_{8}=1 / 6, \quad c_{9}=-3 / 8, \quad c_{10}=0, \\
& c_{11}=0, \quad c_{12}=-1 / 4, \quad c_{13}=1 / 24, \quad c_{14}=1 / 8, \quad c_{15}=1 / 24 \text {. }
\end{aligned}
$$

\section{Partial and Covariant Derivatives of Functions}

For any smooth function $f$ defined on a Kähler manifold, we may derive from (7) the following well-known identities:

$$
f_{/ \alpha}=f_{\alpha}, \quad f_{/ i \bar{j}}=f_{/ \bar{j} i}=f_{i \bar{j}},
$$




$$
\begin{gathered}
f_{/ i j}=f_{i j}-\sum_{k} \Gamma_{i j}^{k} f_{k}, \quad f_{/ \overline{i j}}=f_{\overline{i j}}-\sum_{k} \Gamma_{\overline{i j}}^{\bar{k}} f_{\bar{k}}, \\
f_{/ i \bar{j} k}=f_{/ k \bar{j} i}=f_{i \bar{j} k}-\sum_{l} \Gamma_{i k}^{l} f_{l \bar{j}}, \quad f_{/ i j \bar{k}}=\left(f_{/ i j}\right)_{\bar{k}} .
\end{gathered}
$$

On the other hand, for any partial derivatives $f_{\alpha_{1} \alpha_{2} \ldots \alpha_{m}}$, there exists a canonical tensor, denoted by $D\left(f_{\alpha_{1} \alpha_{2} \ldots \alpha_{m}}\right)$, that coincides with $f_{\alpha_{1} \alpha_{2} \ldots \alpha_{m}}$ at the center of any normal coordinate system. Below we will extend Theorem 4.4 to functions. First we slightly modify the definitions in $\S 4$.

Definition 6.1. Denote by $\mathscr{T}_{f}\left(a_{1} \cdots a_{k} \mid \bar{b}_{1} \cdots \bar{b}_{m}\right)$ the set of all decorated rooted trees $T$ with root $r$ such that all non-root vertices of $T$ are stable and for each directed edge $u v$ with $u, v \neq r$, we have $i(u)<i(v)$.

Define $\mathscr{T}_{f}\left(a_{1} \cdots a_{p}\left|a_{p+1} \cdots a_{k}\right| \bar{b}_{1} \cdots \bar{b}_{m}\right)$ to be the subset of $\mathscr{T}_{f}\left(a_{1} \cdots a_{k} \mid \bar{b}_{1} \cdots \bar{b}_{m}\right)$ containing trees with the property that there does not exist a proper subtree which contains at least two indices from $\left\{a_{1}, \ldots, a_{p}\right\}$.

For each $T \in \mathscr{T}_{f}\left(a_{1} \cdots a_{k} \mid \bar{b}_{1} \cdots \bar{b}_{m}\right)$, we associate a unique monomial of curvature tensors $R_{T}$ given by

$$
R_{T}=(-1)^{|V(T)|-1} \prod_{e \in E(T)} g^{a_{e} \bar{b}_{e}} \prod_{v \in V(T)} R_{v}
$$

where the indices $a_{e}, \bar{b}_{e}$ correspond to the head and tail of $e$ respectively and $R_{v}$ is the curvature tensor obtained by arranging the indices of half-edges of $v$ as follows

$$
R_{v}= \begin{cases}f_{/ a_{*} \cdots a_{*} \bar{b}_{*} \cdots \bar{b}_{*},}, & v=r \\ R_{a_{*} \bar{b}_{i(v)} a_{*} \bar{b}_{e} / a_{*} \cdots a_{*} \bar{b}_{*} \cdots \bar{b}_{*},}, & v \neq r .\end{cases}
$$

Note that when $v \neq r, \bar{b}_{e}$ corresponds to the tail of the unique inward edge $e \in E(T)$ of $v$. By the second Bianchi identity (5) and the Ricci formula (6), $f_{/ a_{*} \cdots a_{*} \bar{b}_{*} \cdots \bar{b}_{*}}$ and $R_{a_{*} \bar{b}_{1} a_{*} \bar{b}_{2} / a_{*} \cdots a_{*} \bar{b}_{*} \cdots \bar{b}_{*}}$ are separately symmetric in the indices $a_{*}$ and $\bar{b}_{*}$, so their orders are irrelevant and thus $R_{T}$ is well-defined.

Lemma 6.2. A tree in $\mathscr{T}_{f}\left(a_{1} \cdots a_{k} \mid \bar{b}_{1} \cdots \bar{b}_{m}\right)$ has at most $\min (k, m)+1$ vertices.

Proof. It is obvious, since all non-root vertices of trees in $\mathscr{T}_{f}\left(a_{1} \cdots a_{k} \mid \bar{b}_{1} \cdots \bar{b}_{m}\right)$ need to be stable. 
Theorem 6.3. Let $k \geq p \geq 0$ and $m \geq 0$. Then

$$
D\left(\left(f_{/ a_{1} \cdots a_{p}}\right)_{a_{p+1} \cdots a_{k} \bar{b}_{3} \cdots \bar{b}_{m}}\right)=\sum_{T \in \mathscr{T}_{f}\left(a_{1} \cdots a_{p}\left|a_{p+1} \cdots a_{k}\right| \bar{b}_{1} \cdots \bar{b}_{m}\right)} R_{T} .
$$

Proof. Obviously (82) holds when $p=k$. From (2), we have

$$
\begin{aligned}
\left(f_{/ a_{1} \cdots a_{p}}\right)_{a_{p+1} \cdots a_{k} \bar{b}_{1} \cdots \bar{b}_{m}}= & \left(f_{/ a_{1} \cdots a_{p} a_{p+1}}\right)_{a_{p+2} \cdots a_{k} \bar{b}_{1} \cdots \bar{b}_{m}} \\
& +\sum_{j=1}^{p}\left(g^{r \bar{s}} g_{a_{p+1} \bar{s} a_{j}} f_{/ a_{1} \cdots a_{j-1} r a_{j+1} \cdots a_{p}}\right)_{a_{p+2} \cdots a_{k} \bar{b}_{1} \cdots \bar{b}_{m}} .
\end{aligned}
$$

Then by induction, in the right-hand side of (83), the first term produces trees with the property that no two of $\left\{a_{1}, \ldots, a_{p}, a_{p+1}\right\}$ are contained in a proper subtree, the last term produces trees with the property that $a_{j}, a_{p+1}(1 \leq j \leq p)$ are contained in a proper subtree. The coefficient of each tree can be determined by exactly the same argument as in the above proof of (61). So we conclude the inductive proof of (82).

Corollary 6.4. Let $k, m \geq 0$. Then

$$
D\left(f_{a_{1} \cdots a_{k} \bar{b}_{1} \cdots \bar{b}_{m}}\right)=\sum_{T \in \mathscr{T}_{f}\left(a_{1} \cdots a_{k} \mid \bar{b}_{1} \cdots \bar{b}_{m}\right)} R_{T} .
$$

Proof. We get the equation by taking $p=0$ in (82).

Example 6.5. Let us compute $D\left(f_{i j \bar{k}}\right)$. Since there is a one-vertex tree and a two-vertex tree in $\mathscr{T}_{f}(i j \mid \bar{k})$, by (84), we get

$$
D\left(f_{i j \bar{k}}\right)=f_{/ i j \bar{k}}-f_{/ l} R_{i \bar{k} j \bar{l}} .
$$

Example 6.6. Let us compute $D\left(f_{i j \bar{k} \bar{l}}\right)$. Besides the one-vertex tree, there are three two-vertex trees in $\mathscr{T}_{f}(i j \mid \bar{k} \bar{l})$,
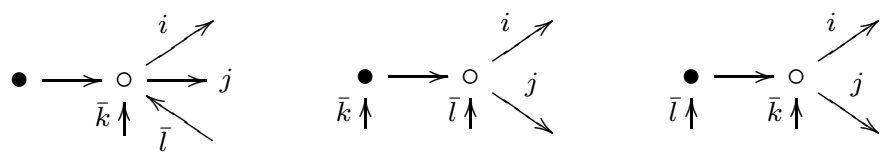

By (84), we get

$$
D\left(f_{i j \bar{k} \bar{l}}\right)=f_{/ i j \bar{k} \bar{l}}-f_{/ p} R_{i \bar{k} j \bar{p} / \bar{l}}-f_{/ p \bar{k}} R_{i \bar{l} j \bar{p}}-f_{/ p \bar{l}} R_{i \bar{k} j \bar{p}} .
$$

Example 6.7. Since there is only one tree in $\mathscr{T}_{f}\left(p_{1} \ldots p_{r}\right)$ and $\mathscr{T}_{f}\left(\bar{p}_{1} \ldots \bar{p}_{r}\right)$, we get $D\left(f_{p_{1} \ldots p_{r}}\right)=f_{/ p_{1} \ldots p_{r}}$ and $D\left(f_{\bar{p}_{1} \ldots \bar{p}_{r}}\right)=f_{/ \bar{p}_{1} \ldots \bar{p}_{r}}$. 


\section{Appendix A. TABles for the COMputation of $D\left(g_{i \bar{i} j \bar{j} k l \bar{k} \bar{l}}\right)$}

Tables 1-6 contains 48 trees with at least two vertices in $D\left(g_{i \bar{i} j \bar{j} k l \bar{k} \bar{l}}\right)$, as demonstrated by Theorem 4.4. The trees are grouped according to the distribution of the external legs. Tables 1-6 are in one-to-one correspondence with the following six types of trees, where the numbers denote the multiplicities of external legs.
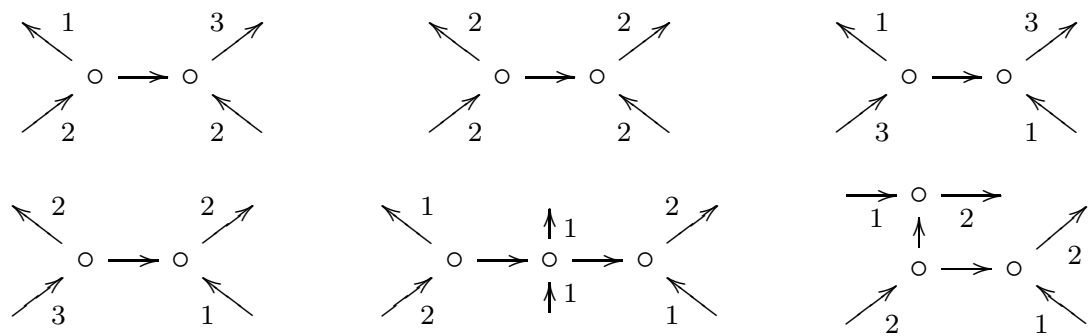

Note that each tree $T$ is weighted by $(-1)^{|V(T)|}$ in the tensor expression. We label a tree by the dagger symbol $\dagger$ if and only if $i, j$ are contained in a proper subtree. These trees add up to give $D\left(\partial_{k l \bar{k} \bar{l}}\left(g^{m \bar{n}} g_{m \bar{i} \bar{j}} g_{i \bar{n} j}\right)\right)$.

TABLE 1

\begin{tabular}{|c|c|c|c|}
\hline$\overbrace{\bar{i} \bar{j}}^{i} \circ \rightarrow 0 \overbrace{\bar{k} \bar{l}}^{j k l}$ & $\overbrace{\bar{i} \bar{j}}^{j} \circ \rightarrow 0 \underset{\bar{k} \bar{l}}{i k l}$ & $\overbrace{\overline{i j}}^{k} \circ \rightarrow 0 \underset{\bar{k} \bar{l}}{i j l}$ & $\overbrace{\overline{i j}}^{l} \circ \rightarrow \circ \underset{\bar{k} \bar{l}}{i j k}$ \\
\hline$\sigma_{9}$ & $\sigma_{9}$ & $\sigma_{10} \dagger$ & $\tilde{\sigma}_{10} \dagger$ \\
\hline
\end{tabular}

TABLE 2

\begin{tabular}{|c|c|c|}
\hline$\overbrace{\overline{i j}}^{i j} \circ \rightarrow 0 \underset{\bar{k} \bar{l}}{k l}$ & $\overbrace{\overline{i j}}^{i k} \circ \rightarrow 0 \underbrace{j l}_{\bar{k} \bar{l}}$ & $\overbrace{\bar{i} \bar{j}}^{i l} \circ \rightarrow 0 \underset{\bar{k} \bar{l}}{j k}$ \\
\hline$\sigma_{11}$ & $\sigma_{12}$ & $\sigma_{12}$ \\
\hline${\underset{\pi}{i j}}_{\pi^{j k}}^{j k} \rightarrow 0 \underset{\bar{k} \bar{l}}{i l}$ & $\overbrace{\overline{i j}}^{j l} \circ \rightarrow 0 \underbrace{i k}_{\bar{k} \bar{l}}$ & $\underset{\overline{i j}}{\pi_{\bar{j}}^{k l}} \circ \rightarrow 0 \underset{\bar{k} \bar{l}}{i j}$ \\
\hline$\sigma_{12}$ & $\sigma_{12}$ & $\sigma_{13} \dagger$ \\
\hline
\end{tabular}


TABLE 3

\begin{tabular}{|c|c|c|c|}
\hline 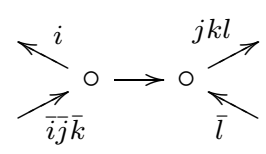 & $\underset{\bar{i} \bar{k} \bar{k}}{j} \circ \rightarrow 0 \frac{i k l}{\bar{l}}$ & $\overbrace{\bar{l}}^{i j l}$ & $\underset{\bar{i} \bar{j} \bar{k}}{l} \circ \rightarrow 0 \underbrace{i j k}_{\bar{l}}$ \\
\hline$\sigma_{12}$ & $\sigma_{12}$ & $\sigma_{12} \dagger$ & $\sigma_{13} \dagger$ \\
\hline$\underset{\bar{i} \bar{j} \bar{l}}{i} \circ \rightarrow \circ \underset{\bar{k}}{\gtrless_{\bar{k}}^{j k l}}$ & 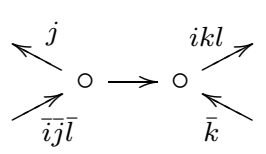 & $\overbrace{\bar{i} \bar{j} \bar{l}}^{k} \circ \rightarrow 0 \frac{\bar{k}}{i j l}$ & $\overbrace{\bar{i} \bar{j} \bar{l}}^{l} \circ \rightarrow 0 \underset{\bar{k}}{i j k}$ \\
\hline$\sigma_{12}$ & $\sigma_{12}$ & $\sigma_{13} \dagger$ & $\sigma_{12} \dagger$ \\
\hline
\end{tabular}

TABLE 4

\begin{tabular}{|c|c|c|c|}
\hline${\underset{\bar{i}}{i \bar{j} \bar{k}}}^{i j} \circ \rightarrow 0 \underbrace{k l}_{\bar{l}}$ & 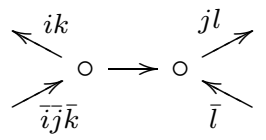 & $\bar{\pi}_{\bar{i} \bar{j} \bar{k}}^{i l}$ & $\underset{\bar{i} \bar{j} \bar{k}}{j k} \circ \rightarrow 0 \frac{i l}{\pi_{\bar{l}}}$ \\
\hline$\widetilde{\sigma}_{9}$ & $\sigma_{9}$ & $\tilde{\sigma}_{10}$ & $\sigma_{9}$ \\
\hline$\underset{\bar{i} \bar{j} \bar{k}}{\mathbb{\pi}^{j l}} \circ \rightarrow 0 \underbrace{i k}_{\bar{l}}$ & $k^{k l} \rightarrow 0^{i j}$ & $r^{i j}$ & $\underset{\bar{i} \bar{j} \bar{l}}{\pi^{i k}} \circ \rightarrow 0 \underset{\bar{k}}{\stackrel{j l}{\pi}}$ \\
\hline$\widetilde{\sigma}_{10}$ & $\sigma_{10} \dagger$ & $\widetilde{\sigma}_{9}$ & $\widetilde{\sigma}_{10}$ \\
\hline 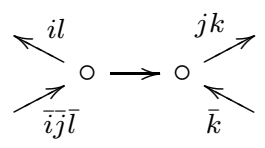 & $\underset{\bar{i} \bar{j} \bar{l}}{\pi^{j k}} \circ \rightarrow 0 \frac{i l}{{ }_{k}}$ & $\mathbb{Z}_{\overline{i j} \bar{l}}^{j l} \circ \rightarrow 0 \frac{i k}{\frac{k}{k}}$ & 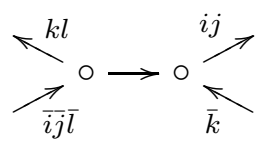 \\
\hline$\sigma_{9}$ & $\widetilde{\sigma}_{10}$ & $\sigma_{9}$ & $\sigma_{10} \dagger$ \\
\hline
\end{tabular}

\section{Appendix B. Enumerations of DeCorated Rooted trees}

Denote by $t_{k, m}(n)$ the number of $n$-vertex trees in $\mathscr{T}_{R}\left(a_{1} \cdots a_{k} \mid \bar{b}_{1} \cdots \bar{b}_{m}\right)$.

Let $s_{p, k, m}(n)$ and $s_{p, k, m}^{c}(n)$ denote respectively the numbers of $n$-vertex trees in $\mathscr{T}_{R}\left(a_{1} \cdots a_{p}\left|a_{p+1} \cdots a_{k}\right| \bar{b}_{1} \cdots \bar{b}_{m}\right)$ and $\mathscr{T}_{R}^{c}\left(a_{1} \cdots a_{p}\left|a_{p+1} \cdots a_{k}\right| \bar{b}_{1} \cdots \bar{b}_{m}\right)$. Namely $s_{p, k, m}(n)$ counts trees with the property that there does not exist a proper subtree which contains at least two indices from $\left\{a_{1}, \ldots, a_{p}\right\}$.

In order to enumerate the number of $n$-vertex decorated trees, we need to count all admissible decorations of inward external legs and outward external legs separately for each $n$-vertex rooted tree, i.e. each vertex should be stable and $i(u)<i(v)$ for any directed edge $u v$ (see Definition 4.3). 
TABLE 5

\begin{tabular}{|c|c|c|}
\hline 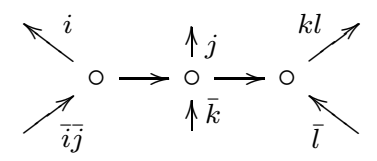 & 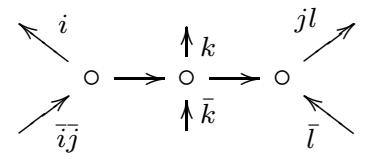 & 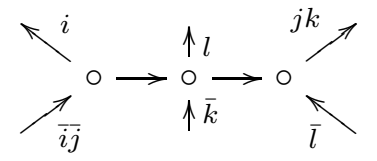 \\
\hline$\sigma_{4}$ & $\sigma_{6}$ & $\sigma_{5}$ \\
\hline 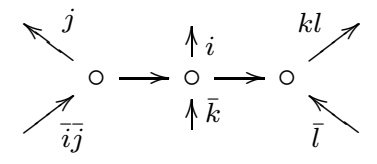 & 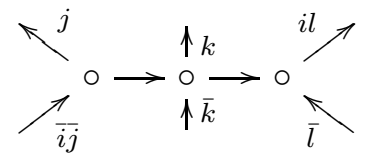 & 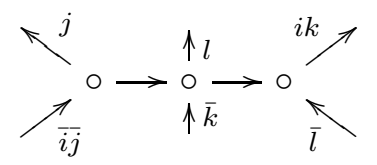 \\
\hline$\sigma_{4}$ & $\sigma_{6}$ & $\sigma_{5}$ \\
\hline 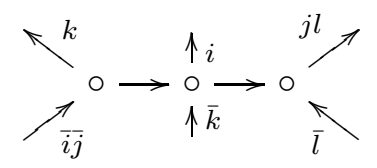 & 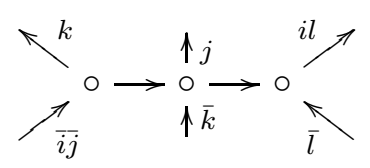 & 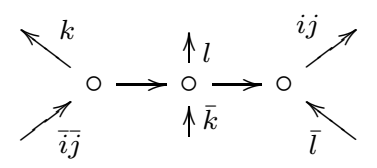 \\
\hline$\sigma_{5} \dagger$ & $\sigma_{5} \dagger$ & $\sigma_{15} \dagger$ \\
\hline 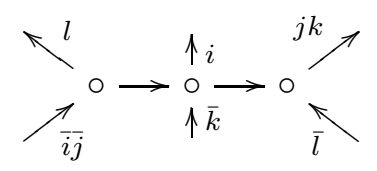 & 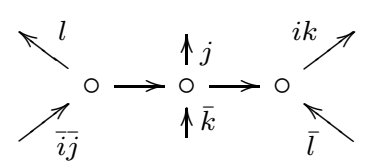 & 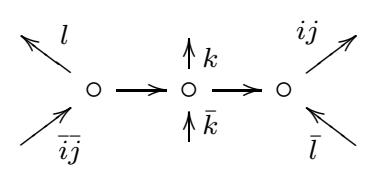 \\
\hline$\sigma_{7} \dagger$ & $\overline{\sigma_{7} \dagger}$ & $\sigma_{5} \dagger$ \\
\hline
\end{tabular}

TABLE 6

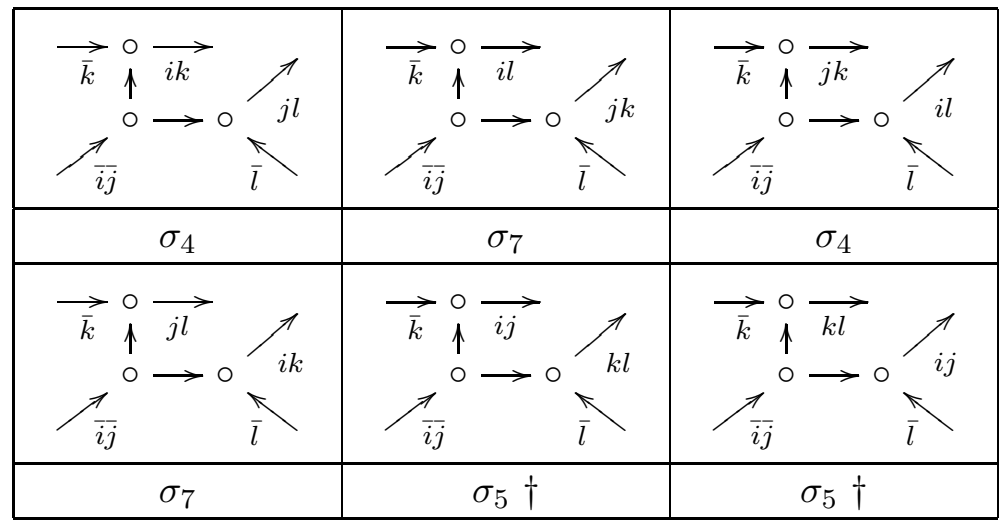

Lemma B.1. Let $k \geq p \geq 2, m \geq 2$.

(i) $t_{k, m}(n)=0$ when $n \geq \min (k, m)$, i.e. a tree in $\mathscr{T}_{R}\left(a_{1} \cdots a_{k} \mid \bar{b}_{1} \cdots \bar{b}_{m}\right)$ has at most $\min (k, m)-1$ vertices. 
(ii) For 2-vertex trees, we have $t_{k, m}(2)=\left(2^{k}-2-k\right)\left(2^{m-2}-1\right)$ and

$$
\begin{aligned}
& s_{p, k, m}(2)=\left((p+1) 2^{k-p}-k-1\right)\left(2^{m-2}-1\right), \\
& s_{p, k, m}^{c}(2)=\left(2^{k}-(p+1) 2^{k-p}-1\right)\left(2^{m-2}-1\right) .
\end{aligned}
$$

(ii) For 3-vertex trees, we have

$$
\begin{gathered}
t_{k, m}(3)=\left(2 \cdot 3^{k}-\frac{3 k}{2} \cdot 2^{k}-5 \cdot 2^{k}+k^{2}+3 k+4\right)\left(\frac{1}{18} 3^{m}-\frac{1}{4} 2^{m}+\frac{1}{2}\right), \\
s_{p, k, m}(3)=\left(3^{k-p}\left(p^{2}+3 p+2\right)-2^{k-p-1}\left(3 k p+3 k+p^{2}+7 p+8\right)+k^{2}+2 k+2\right) \\
\times\left(\frac{1}{18} 3^{m}-\frac{1}{4} 2^{m}+\frac{1}{2}\right) .
\end{gathered}
$$

(iii) For 4-vertex trees, we have

$$
\begin{gathered}
t_{k, m}(4)=\left(6 \cdot 4^{k}-3^{k}(4 k+19)+2^{k}\left(\frac{7}{4} k^{2}+\frac{43}{4} k+21\right)-\left(k^{3}+4 k^{2}+9 k+9\right)\right) \\
\times\left(\frac{1}{96} 4^{m}-\frac{1}{18} 3^{m}+\frac{1}{8} 2^{m}-\frac{1}{6}\right) .
\end{gathered}
$$

Proof. (i) is obvious, since each tree in $\mathscr{T}_{R}\left(a_{1} \cdots a_{k} \mid \bar{b}_{1} \cdots \bar{b}_{m}\right)$ is stable.

Now we prove (ii). From the unique one 2-vertex tree, we have

$$
\begin{aligned}
t_{k, m}(2) & =\sum_{i=2}^{k-1}\left(\begin{array}{c}
k \\
i
\end{array}\right) \sum_{j=1}^{m-2}\left(\begin{array}{c}
m-2 \\
j
\end{array}\right)=\left(2^{k}-2-k\right)\left(2^{m-2}-1\right), \\
s_{p, k, m}(2) & =\left(\sum_{i=2}^{k-p}\left(\begin{array}{c}
k-p \\
i
\end{array}\right)+p \sum_{i=1}^{k-p}\left(\begin{array}{c}
k-p \\
i
\end{array}\right)\right) \sum_{j=1}^{m-2}\left(\begin{array}{c}
m-2 \\
j
\end{array}\right) \\
& =\left((p+1) 2^{k-p}-k-1\right)\left(2^{m-2}-1\right), \\
s_{p, k, m}^{c}(2) & =\left(\begin{array}{c}
\sum_{i=2}^{p}\left(\begin{array}{c}
p \\
i
\end{array}\right) \sum_{j=0}^{k-p}\left(\begin{array}{c}
k-p \\
j
\end{array}\right)-1
\end{array}\right) \sum_{j=1}^{m-2}\left(\begin{array}{c}
m-2 \\
j
\end{array}\right) \\
& =\left(2^{k}-(p+1) 2^{k-p}-1\right)\left(2^{m-2}-1\right) .
\end{aligned}
$$

Next we prove (iii). There are two 3 -vertex rooted trees

$$
\stackrel{\circ}{x} \rightarrow \stackrel{\circ}{y} \rightarrow \stackrel{\circ}{z} \quad \stackrel{\circ}{u} \longleftarrow \stackrel{\circ}{v} \rightarrow \stackrel{\circ}{w}
$$


First we count the number of admissible decorations of inward external legs for the two trees, which respectively equal to

$$
\begin{gathered}
\sum_{i=0}^{m-4}\left(\begin{array}{c}
m-2 \\
i
\end{array}\right) \sum_{j=0}^{m-4-i}\left(\begin{array}{c}
m-3-i \\
j
\end{array}\right)=\frac{1}{18} 3^{m}-\frac{1}{4} 2^{m}+\frac{1}{2} \\
\frac{1}{2} \sum_{i=0}^{m-4}\left(\begin{array}{c}
m-2 \\
i
\end{array}\right) \sum_{j=1}^{m-3-i}\left(\begin{array}{c}
m-2-i \\
j
\end{array}\right)=\frac{1}{18} 3^{m}-\frac{1}{4} 2^{m}+\frac{1}{2}
\end{gathered}
$$

We count the number of all admissible decorations of outward external legs $\left(\operatorname{deg}^{+}(v) \geq 2\right.$ for all vertices) to the two 3 -vertex trees of $(87)$.

$$
\begin{aligned}
\sum_{i=0}^{k-2}\left(\begin{array}{l}
k \\
i
\end{array}\right) \sum_{j=1}^{k-i-1}\left(\begin{array}{c}
k-i \\
j
\end{array}\right)+\sum_{i=0}^{k-4}\left(\begin{array}{c}
k \\
i
\end{array}\right) \sum_{j=2}^{k-i-2}\left(\begin{array}{c}
k-i \\
j
\end{array}\right) \\
=2 \cdot 3^{k}-\frac{3 k}{2} \cdot 2^{k}-5 \cdot 2^{k}+k^{2}+3 k+4
\end{aligned}
$$

which gives the formula of $t_{k, m}(3)$.

We count the number of all admissible decorations of outward external legs to the two 3-vertex trees of (87), such that there does not exist a proper subtree which contains at least two indices from $\left\{a_{1}, \ldots, a_{p}\right\}$. We call $\left\{a_{1}, \ldots, a_{p}\right\}$ the set of special indices. The following summation may be simplified to get the first factor in the formula of $s_{p, k, m}(3)$.

$$
\begin{aligned}
& \sum_{i=0}^{k-p-3}\left(\begin{array}{c}
k-p \\
i
\end{array}\right) \sum_{j=1}^{k-p-i-2}\left(\begin{array}{c}
k-p-i \\
j
\end{array}\right)+p \sum_{i=0}^{k-p-2}\left(\begin{array}{c}
k-p \\
i
\end{array}\right) \sum_{j=0}^{k-p-i-2}\left(\begin{array}{c}
k-p-i \\
j
\end{array}\right) \\
+ & p \sum_{i=0}^{k-p-2}\left(\begin{array}{c}
k-p \\
i
\end{array}\right) \sum_{j=1}^{k-p-i-1}\left(\begin{array}{c}
k-p-i \\
j
\end{array}\right)+\sum_{i=0}^{k-p-4}\left(\begin{array}{c}
k-p \\
i
\end{array}\right) \sum_{j=2}^{k-p-i-2}\left(\begin{array}{c}
k-p-i \\
j
\end{array}\right) \\
+ & 2 p \sum_{i=0}^{k-p-3}\left(\begin{array}{c}
k-p \\
i
\end{array}\right) \sum_{j=1}^{k-p-i-2}\left(\begin{array}{c}
k-p-i \\
j
\end{array}\right)+2\left(\begin{array}{c}
p \\
2
\end{array}\right) \sum_{i=0}^{k-p-2}\left(\begin{array}{c}
k-p \\
i
\end{array}\right) \sum_{j=1}^{k-p-i-1}\left(\begin{array}{c}
k-p-i \\
j
\end{array}\right)
\end{aligned}
$$

The first three terms come from the first tree of (87) corresponding to the three cases: (i) all special indices belong to $x$; (ii) all but one special indices belong to $x$ and one special index belongs to $y$; (iii) all but one special indices belong to $x$ and one special index belongs to $z$. 
The last three terms come from the second tree of (87) corresponding to the three cases: (i) all special indices belong to $v$; (ii) all but one special indices belong to $v$ and one special index belongs to $u$ or $w$; (iii) all but two special indices belong to $v$, one special index belongs to $u$ and one special index belongs to $w$.

The proof of the formula for $t_{k, m}(4)$ is similar to the above. We need to deal with the four 4-vertex rooted trees in Figure 1.

\section{REFERENCES}

[1] S. Alexakis, The decomposition of global conformal invariants, Annals of Mathematics Studies, 182. Princeton University Press, Princeton, NJ, 2012.

[2] C. Arezzo, A. Loi and F. Zuddas, On homothetic balanced metrics, Ann. Glob. Anal. Geom. 41 (2012), 473-491.

[3] T. Bailey, M. Eastwood and C. Graham, Invariant theory for conformal and CR geometry, Ann. of Math., 139 (1994), 491-552.

[4] D. Barrett and L. Lee, On the Szegö Metric, J. Geom. Anal. (to appear).

[5] F. A. Berezin, Quantization, Math. USSR Izvest. 8 (1974), 1109-1163.

[6] R. Berman, B. Berndtsson and J. Sjöstrand, A direct approach to Bergman kernel asymptotics for positive line bundles, Ark. Mat. 46 (2008), 197-217.

[7] D. Boichu and G. Coeuré, Sur le noyau de Bergman des domaines de Reinhardt, Invent. Math. 72 (1983), 131-152.

[8] L. Boutet de Monvel and J. Sjöstrand, Sur la singularité des noyau de Bergman et de Szegö, Astérisque, 34-35 (1976), 123-164.

[9] D. Catlin, The Bergman kernel and a theorem of Tian, Analysis and geometry in several complex variables (Katata, 1997), 1999, 1-23.

[10] L. Charles, Berezin-Toeplitz operators, a semi-classical approach, Comm. Math. Phys. 239 (2003), 1-28.

[11] S.-Y. Cheng and S.-T. Yau, On the existence of a complete Kähler metric on non-compact complex manifolds and the regularity of Fefferman's equation, Comm. Pure Appl. Math. 33 (1980), 507-544.

[12] S. S. Chern and J. Moser, Real hypersurfaces in complex manifolds, Acta Math. 133 (1974), 219-271.

[13] X. Dai, K. Liu and X. Ma, On the asymptotic expansion of Bergman kernel, J. Differential Geom. 72 (2006), no. 1, 1-41.

[14] X. Dai and K. Liu and X. Ma, A remark on weighted Bergman kernels on orbifolds, Math. Res. Lett. 19 (2012), 143-148. 
[15] S. Donaldson, Scalar curvature and projective embeddings, I, J. Differential Geom. 59 (2001), no. 3, 479-522.

[16] M. Douglas and S. Klevtsov, Bergman Kernel from Path Integral, Comm. Math. Phys. 293 (2010), 205-230.

[17] M. Engliš, The asymptotics of a Laplace integral on a Kähler manifold, J. Reine Angew. Math. 528 (2000), 1-39.

[18] M. Engliš, A Forelli-Rudin construction and asymptotics of weighted Bergman kernels, J. Funct. Anal. 177 (2000), 257-281.

[19] M. Engliš and H. Xu, Forelli-Rudin construction and asymptotic expansion of Szegö kernel, preprint.

[20] C. Fefferman, The Bergman kernel and biholomorphic mappings of pseudoconvex domains, Invent. Math. 26 (1974), 1-65.

[21] C. Fefferman, Monge-Ampère equations, the Bergman kernel and geometry of pseudoconvex domains, Ann. of Math. 103 (1976), 395-416; Correction, ibid., 104 (1976), 393-394.

[22] C. Fefferman, Parabolic invariant theory in complex analysis, Adv. Math. 31 (1979), 131262.

[23] S. Fu and B. Wong, On strictly pseudoconvex domains with Kähler-Einstein Bergman metrics, Math. Res. Lett. 4 (1997), 697-703.

[24] M. García-Fernández, J. Ross, Limits of balanced metrics on vector bundles and polarised manifolds, arXiv:1111.2819.

[25] C. R. Graham, Scalar boundary invariants and the Bergman kernel, in "Complex Analysis II", Lecture Notes in Math., 1276, pp. 108-135, Springer, 1987.

[26] C. R. Graham, Higher asymptotics of the complex Monge-Ampère equation, Compositio Math. 64 (1987), 133-155.

[27] K. Hirachi, Construction of boundary invariants and the logarithmic singularity of the Bergman kernel, Ann. of Math. 151 (2000), 151-190.

[28] K. Hirachi, CR invariants of weight 6, Several complex variables (Seoul, 1998), J. Korean Math. Soc. 37 (2000), 177-191.

[29] K. Hirachi and G. Komatsu, Invariant theory of the Bergman kernel, in "CR-geometry and overdetermined systems" (Osaka, 1994), 167-220, Adv. Stud. Pure Math., 25, Math. Soc. Japan, Tokyo, 1997.

[30] K. Hirachi, G. Komatsu and N. Nakazawa, Two methods of determining local invariants in the Szegö kernel, in "Complex Geometry", Lecture Notes in Pure and Appl. Math., 143, pp. 77-96, Dekker, 1993.

[31] K. Hirachi, G. Komatsu and N. Nakazawa, CR invariants of weight five in the Bergman kernel, Adv. Math. 143 (1999), 185-250.

[32] L. Hörmander, $L^{2}$ estimates and existence theorems for the $\bar{\partial}$ operator, Acta Math. 113 (1965), 89-152.

[33] C-Y. Hsiao and G. Marinescu, Asymptotics of spectral function of lower energy forms and Bergman kernel of semi-positive and big line bundles, arXiv:1112.5464.

[34] J. Kamimoto, Newton polyhedra and the Bergman kernel, Math. Z. 246 (2004), 405-440. 
[35] A. Karabegov and M. Schlichenmaier, Identification of Berezin-Toeplitz deformation quantization, J. Reine Angew. Math. 540 (2001), 49-76.

[36] M. Kuranishi, The formula for the singularity of Szegö kernel. I, J. Korean Math. Soc. 40 (2003), 641-666.

[37] J. Lee and R. Melrose, Boundary behaviour of the complex Monge-Ampère equation, Acta Math. 148 (1982), 159-192.

[38] V. Liskovec, On a recurrence method of counting graphs with labelled vertices, Soviet Math. Dokl. 10 (1969), 242-246.

[39] K. Liu, Heat kernel and moduli spaces, Math. Res. Lett. 3 (1996), 743-762.

[40] K. Liu, Heat kernel and moduli spaces II., Math. Res. Lett. 4 (1997), 569-588.

[41] K. Liu, Heat kernels, symplectic geometry, moduli spaces and finite groups., Surv. Differ. Geom. 5 (1999), 527-542.

[42] C. Liu and Z. Lu, On the asymptotic expansion of Tian-Yau-Zelditch, arixv:1105.0221.

[43] A. Loi, The Tian-Yau-Zelditch asymptotic expansion for real analytic Kähler metrics, Int. J. Geom. Methods in Modern Phys. 1 (2004), 253-263.

[44] A. Loi, A Laplace integral on a Kähler manifold and Calabi's diastasis function, Differential Geom. Appl. 23 (2005), 55-66.

[45] Z. Lu, On the lower order terms of the asymptotic expansion of Tian-Yau-Zelditch, Amer. J. Math. 122 (2000), no. 2, 235-273.

[46] X. Ma and G. Marinescu, Generalized Bergman kernels on symplectic manifolds, Adv. Math. 217 (2008), no. 4, 1756-1815.

[47] N. Mok and S.-T. Yau, Completeness of the Kähler-Einstein metric on bounded domains and the characterization of domains of holomorphy by curvature conditions, The Mathematical Heritage of Henri Poincaré, Proc. Sympos. Pure Math., 39, Amer. Math. Soc., 1983, pp. 41-59.

[48] N. Nakazawa, Asymptotic expansion of the Bergman kernel for strictly pseudoconvex complete Reinhardt domains in $\mathbb{C}^{2}$, Osaka J. Math. 31 (1994), 291-329.

[49] J. Ross and R. Thomas, Weighted Bergman kernels on orbifolds, J. Differential Geom. 88 (2011), 87-107.

[50] M. Schlichenmaier, Berezin-Toeplitz Quantization and Star Products for Compact Kähler Manifolds, arXiv:1202.5927.

[51] H. Xu, A closed formula for the asymptotic expansion of the Bergman kernel, Comm. Math. Phys. 314 (2012), 555-585.

[52] H. Xu, An explicit formula for the Berezin star product, Lett. Math. Phys. 101 (2012), 239-264.

[53] H. Xu, Weyl invariant polynomial and deformation quantization on Kähler manifolds, J. Geom. Phys. (to appear).

[54] H. Xu and S.-T. Yau, Trees and tensors on Kähler manifolds, Ann. Global Anal. Geom. 44 (2013), 151-168.

[55] S.-T. Yau, Problem Section, Seminar on Differential geometry (S.-T. Yau, eds.), Ann. of Math. Studies, vol. 102, Princeton Univ. Press, 1982, pp. 669-706.

[56] S.-T. Yau, Nonlinear analysis in geometry, L'Énseignement Math. 33 (1987), 109-158. 
[57] S. Zelditch, Szegö kernel and a theorem of Tian, Internat. Math. Res. Notices, 1998, no. 6, 317-331.

[58] S. Zelditch, Survey of the inverse spectral problem, Surv. Differ. Geom. IX (2004) 401-467.

\section{Hao Xu}

Center of Mathematical Sciences, Zhejiang University, Hangzhou, Zhejiang 310027, China;

Department of Mathematics, Harvard University, Cambridge, MA 02138, USA

E-mail: mathxuhao@gmail.com 